\title{
Current analytical methods for plant auxin quantification - A Review
}

Sara Porfírio ${ }^{1,3^{\star}}$, Marco D. R. Gomes da Silva ${ }^{2^{\star}}$, Augusto Peixe ${ }^{1}$, Maria J. Cabrita ${ }^{1}$, Parastoo Azadi $^{3}$

${ }^{1}$ Instituto de Ciências Agrárias e Ambientais Mediterrânicas - ICAAM, Universidade de Évora, 7006-554 Évora, Portugal

2 LAQV, REQUIMTE, Departamento de Química, Faculdade de Ciências e Tecnologia, Universidade Nova de Lisboa, 2829-516 Caparica, Portugal

${ }^{3}$ Complex Carbohydrate Research Center, The University of Georgia, 315 Riverbend Road, Athens, Georgia 30602, USA

\section{Corresponding authors}

*E-mail:mdr@fct.unl.pt

Phone: +351212948351

*E-mail: sporfirio@uevora.pt (or porfirio@uga.edu)

Phone: +351266 760869

(C) 2015. This manuscript version is made available under the Elsevier user license http://www.elsevier.com/open-access/userlicense/1.0/ 


\section{Abstract}

Plant hormones, and especially auxins, are low molecular weight compounds highly involved in the control of plant growth and development. Auxins are also broadly used in horticulture, as part of vegetative plant propagation protocols, allowing the cloning of genotypes of interest. Over the years, large efforts have been put in the development of more sensitive and precise methods of analysis and quantification of plant hormone levels in plant tissues. Although analytical techniques have evolved, and new methods have been implemented, sample preparation is still the limiting step of auxin analysis. In this review, the current methods of auxin analysis are discussed. Sample preparation procedures, including extraction, purification and derivatization, are reviewed and compared. The different analytical techniques, ranging from chromatographic and mass spectrometry methods to immunoassays and electrokinetic methods, as well as other types of detection are also discussed. Considering that auxin analysis mirrors the evolution in analytical chemistry, the number of publications describing new and/or improved methods is always increasing and we considered appropriate to update the available information. For that reason, this article aims to review the current advances in auxin analysis, and thus only reports from the past 15 years will be covered.

Keywords: Plant hormones, Auxins quantification, Sample preparation, Chromatographic analysis, Mass spectrometry, Immunoassays 
1

2

3

4

5

6

7

8

\section{Introduction}

Plant hormones are a group of structurally diverse compounds which regulate most processes involved in plant growth and development [1,2]. Auxins are by far the most studied group of plant hormones mainly because they were the first to be discovered [3,4], and because they are widely used in plant propagation protocols [5-9], given their role in adventitious root formation in different species [10,11].

Although there are several compounds with auxin activity, indole-3-acetic acid (IAA) is by far the most physiologically important plant hormone. In fact, it is surprising how such a small molecule can influence so many different processes. IAA has been shown to be involved in many aspects of plant growth and development: cell elongation, regulation of apical dominance, vascular differentiation, fruit development, lateral and adventitious root formation [2]. Indeed, IAA has long been considered "the growth hormone" $[4,12]$.

The widespread use of auxins in plant propagation protocols and physiological studies $[9,13]$, has led to many efforts towards the development of analytical methods for the quantification of the very low auxin levels in plants. The goal of this review is to summarize the recent advances (since 2000) in analytical methods for the quantification of two naturally occurring auxins, IAA and indole-3-butyric acid (IBA) in plant tissues.

\section{Analytical methods for auxin quantification}

Auxins are indolic acids distinguishable by a variable side chain (see Fig.1). One of the main obstacles to auxin quantification is the low endogenous concentration of analyte present in plant samples. Like any plant hormone, auxins are typically found in trace amounts in plant tissues, usually at the ppb level $-0.1-50 \mathrm{ng} \mathrm{g}^{-1} \mathrm{FW}[14,15]$, making the qualitative and quantitative analysis of these compounds very difficult [16]. The analysis is further hindered by the high amount of interfering substances contained in crude plant extracts [17]. However, the main difficulty associated with auxin quantification may be the low yield frequently obtained as a result of oxidation processes and the tendency of indolic compounds to bind irreversibly to glass [18]. Nevertheless, these effects can be compensated by the use isotope of dilution 
techniques (described in detail in [18]). Stable isotope-labeled compounds are very good internal standards on account of their physical and chemical similarities with the original analytes, providing correction for analyte loss and ion suppression by co-eluting substances $[16,17]$. Structural similarities between analytes and internal standards entail an identical or nearly identical behavior during extraction and chromatographic separation, yet the difference in mass allows them to be distinguished by mass spectrometry (MS) [17]. Nevertheless, it should be noted that the mass difference between analyte and internal standard must be enough to avoid isotopic interference [19], which is why $\left[{ }^{13} C_{6}\right]$ IAA is the best internal standard for IAA quantification: the incorporation of $\operatorname{six}{ }^{13} \mathrm{C}$ atoms in the benzene ring of the indole group provides a mass difference of 6 units between analyte and internal standard. In the case of IAA, different types of isotopically-labeled standards are commercially available, but this is not so for other auxins. To quantify IBA, for example, proper internal standards (such as $\left[{ }^{13} \mathrm{C}_{8},{ }^{15} \mathrm{~N}_{1}\right]$ IBA) have to be synthesized, as reported by some authors [20], which brings an extra workload. Alternatively, other compounds can be used as internal standards provided they are closely related to the target compounds in terms of physicochemical properties and stability, and are not naturally produced by the plant or are produced in undetectable amounts [17].

Considering the above, the development of extremely sensitive and selective analytical methods is crucial for the accurate quantification of plant hormones, considering that most current studies require increasingly smaller amounts of plant material and faster analyses. Many methods have been developed for the simultaneous quantification of several plant hormones (Fig. 2) [21-25], however, until recently, a rapid, sensitive, accurate and efficient standard method was still needed for faster progress in botany research [14].

\subsection{Sample preparation}

Despite the advances in analytical methods in the past decades, and with the exception of microtechniques $[20,26]$, sample preparation is still the major step in auxin quantification, taking up to $80 \%$ of the total time of analysis [27]. Depending on the type of plant material and the method used, the complete process of sample preparation can involve sample homogenization, 
extraction of analytes from the matrix and purification of the extract to remove co-extracted interfering substances (extract enrichment) [16].

Sample collection is the first of a series of key steps in the preparation of samples prior to analysis. It is very important to work fast and collect the samples in a way that avoids changes in hormone levels induced by wounding [28]. One way of doing so involves flash-freezing the samples in liquid nitrogen when they are collected from the plant, a step particularly important when dealing with large sample amounts ( $\geq 50 \mathrm{mg}$ ). In this case, the next crucial step involves grinding the frozen samples, which can also be done in liquid nitrogen to prevent defrosting of the sample and chemical degradation of auxins [14]. However, if a small amount of sample is used (few mg) grinding should be bypassed to avoid sample loss. Instead, tissues may be disrupted by ceramic beads in a tissue homogenizer [22] or homogenized directly with extraction buffer in vibrating-ball micromills [21].

Another option involves freeze-drying the samples before grinding, which eliminates time constraints related to the possibility of defrosting and minimizes chemical degradation of analytes. Actually it has been shown that freshly frozen and freeze-dried plant tissues do not differ in plant hormone contents [29]. However, it should be mentioned that freeze-drying is not suitable for all types of plant tissues, so the method used in sample preparation should be chosen based on the type of plant material.

\subsubsection{Extraction}

Because plant samples are in solid form, the first step of any analytical protocol is a classical solid-liquid extraction that will extract the analytes into a liquid phase, which can be used for further purification and concentration steps.

Extraction yield is highly dependent on the choice of the right extraction solvent, which frequently is a mixture rather than an individual solvent. An ideal solvent would extract the maximum amount of auxins and the minimum amount of matrix components, but since the interfering matrix is in large excess over auxins, is very difficult to find such a solvent. 
Auxins are only slightly soluble in water, and highly soluble in organic solvents (e.g. methanol, ethanol, acetone, diethyl ether and dimethyl sulfoxide) or in aqueous alkaline solutions such as basic buffers [30].

Many different solvents have been applied in auxin extraction: methanol [21,31,32], methanol : water [33-35], acetone : water [36], methanol : $\mathrm{KH}_{2} \mathrm{PO}_{4}$ buffer [37], isopropanol : $\mathrm{H}_{2} \mathrm{O}: \mathrm{HCl}$ [28], isopropanol : imidazole buffer $[18,38]$. There are also some references to the use of aqueous buffers (phosphate buffer $\mathrm{pH}$ 6.5) [39] and, in an attempt to use more environment-friendly extraction solvents, several ionic liquids were tested as extraction solvents of IBA from pea samples [40]. Although good results were obtained with 1-butyl-3-methylimidazolium hexafluorophosphate $\left(\left[\mathrm{C}_{4} \mathrm{mim}\right]\left[\mathrm{PF}_{6}\right]\right)$, a previous extraction step using phosphate buffer is still required [40]. Among these different mixtures, methanol has become the most popular solvent for extraction of plant hormones possibly because it easily penetrates plant cells during extraction due to its low molecular weight and high polarity ([15] and references therein). Nevertheless, auxin extraction with primary alcohols can possibly result in the esterification of IAA [18], which should be taken into account when choosing an analytical protocol. To avoid this type of artifacts, secondary alcohols such as isopropanol or solvents with similar polarity, such as acetonitrile can be used instead $[18,41]$.

The choice of extraction solvent also should be influenced by the analytical technique to be used. Recently, Novák et al. [42] showed that organic solvents may be unsuitable for LC/MRM/MS analysis. When comparing the performance of $80 \%$ methanol, $70 \%$ acetone and 2-isopropanol/Na-phosphate buffer pH 7.0 (2:3), unbuffered organic solvents extracted a much higher concentration of interfering compounds such as lipids and pigments. However, phosphate buffers have been suggested to cause enzymatic degradation of auxins during extraction, and acetone is reported to produce lower recoveries than methanol and acetonitrile [43].

Auxins are easily oxidized and degraded by exposure to light, oxygen and high temperatures [30]. Although this is less of a problem when working at the microscale, if the sample preparation procedure is long, which is usually associated with large sample sizes and bulk extractions, an antioxidant can be added to the extraction solvent to prevent auxin degradation. 
113 The most widely used antioxidants are butylated hydroxytoluene (BHT) $[33-35,39]$ and diethyl 114 dithiocarbamate [36,44]. In such cases, considering the reasons above, extraction is normally 115 carried out for several hours at low temperature. It should be mentioned, however, that such 116 additives interfere with subsequent analysis and their use can and should be avoided if rapid 117 analysis methods are to be used. Extraction efficiency can be improved using microwave energy (microwave-assisted extraction (MAE)), which also speeds up the whole procedure. However, the high temperatures produced by microwaves can destroy some plant compounds [45]. To overcome this problem, extraction can be performed under vacuum conditions. This procedure not only prevents oxidation of analytes, but also allows extraction to be performed at low temperatures preventing thermal degradation. An example of this procedure was described by Hu et al. [46] who used vacuum microwave-assisted extraction (VMAE) to extract IAA and IBA from pea and rice seeds.

As previously mentioned, depending on the type of plant material and technique used, further sample clean-up may be still necessary between extraction and analysis. While this type of procedures is losing significance in most modern protocols [26], sample purification is still very important to remove interferents and increase sensitivity of the analytical methods when working with bulk extractions.

\subsubsection{Purification and clean-up}

132 Sample purification can be crucial for a successful analysis because it isolates the analytes of 133 interest from their matrix constituents, while cleaning the sample. This procedure not only improves separation and detection by the analytical methods used, but also reduces the cost of analysis by increasing the instrument's maintenance interval [27]. However, the type of plant tissue and the available instrumentation will greatly influence the need for purification methods.

137 When working with small amounts (a few mg or even less) of herbaceous tissues and having access to powerful instrumentation such as high-resolution MS, sample clean-up becomes less important and can even be detrimental. Nevertheless, auxin quantification is frequently 
performed in more ligneous tissues using less powerful instrumentation. In these situations, purification of crude extracts still is a fundamental step of sample preparation.

\subsubsection{Adaptations of liquid-liquid extraction (LLE) and solid-phase extraction (SPE)}

Classical techniques such as LLE and SPE are by far the most used methods of purification in auxin analysis (see Tables S1-S4). Given their simplicity and the possibility of customization and automation, they became the preferred purification techniques for most analytes [47], although SPE has been associated with higher recoveries than LLE [48]. Particularly, the purification of IAA by C18-SPE has been optimized in detail as part of analytical protocols starting from samples extracted with $80 \%$ methanol [49]. Ion exchange chromatography (IEC) has also been applied as a purification step in combination with SPE and/or LLE. For example, DEAE columns have been combined with C18 SPE cartridges [50,51], or with LLE [52] or even with other IEC columns [53] (Tables S1 and S2). In other cases, a dual-mode SPE purification step including ion exchange columns (Oasis MCX) in combination with $\mathrm{C} 18$ cartridges was used to isolate IAA from other plant hormones [54,55]. Mixed-mode cation-exchange cartridges such as Oasis MCX can improve detection by LC/ESI-MS/MS by reducing the matrix effect through the selective retention of interferents, like pigments and lipids [56]. Further improvements in analyte recovery can be achieved by combining SPE with LLE in the same protocol, as described by Cui et al. [48], who performed a comparative study on the performance of different SPE cartridges (Oasis HLB, HyperSep C18, Oasis MAX and Oasis MCX) and LLE solvents (ethyl acetate, hexane and dichloromethane). The authors concluded that Oasis MCX cartridges combined with ethyl acetate LLE was the best combination to extract auxins (among other plant hormones) from two-month-old leaves of oilseed rape.

Nevertheless, the relatively large amount of sample needed (frequently hundreds of $\mathrm{mg}$ ), the high solvent waste produced, as well as the length of operation time associated with both LLE and SPE methods, have stimulated the development of microextraction techniques, such as SPME and dispersive liquid-liquid microextraction (DLLME), which consume minimal volumes of 
mentioning the work of Liu et al. [20] who developed a miniaturized SPE protocol for auxin isolation from plant tissues. These authors developed a high-throughput purification protocol based on SPE TopTips for the quantification of IBA, IAA and IAA precursors by GC/MS/MS using less than $20 \mathrm{mg}$ of tissue. The protocol, successfully applied to Arabidopsis and tomato tissues, not only minimizes the volume of solvents used (overcoming the main disadvantage of SPE) but also can be customized based on the choice of SPE resin. A similar approach had previously been developed by Müller et al. [21], but in this case the protocol was designed for the isolation of multiple classes of plant hormones, including IAA, from Arabidopsis tissues (20 $200 \mathrm{mg} \mathrm{FW}$ ). Other approaches used SPME to extract IAA and IBA from xylem fluids and foliage material of Musa basjoo and Viola baoshanensis, respectively [37], and carbowaxcoated fibers were more efficient than polyacrylate fibers. Although the method was successfully applied to both types of samples, it was more efficient when applied to the xylem fluid as no matrix effect was found in this case, which narrows the application field of the method. Indeed, SPME is only seldom used in auxin extraction. Other constraints for this technique include the limited number of commercially available fiber coatings [37] and the requirement for volatile or semi-volatile analytes [58]. For instance, polydimethylsiloxane fibers have been used for SPME extraction of methyl jasmonate [58,59], however they were not useful in the extraction of its non-volatile form, jasmonic acid [60]. Nevertheless, a polyaniline nanofiber was recently developed for in vivo SPME detection of three acidic plant hormones, which did not include auxins [61].

Further adaptations of SPE include the application of MIPs as SPE sorbents [62] in a process called molecularly imprinted SPE (MISPE) (for reviews see [63-65]). MIPs are tailor-made polymeric materials designed for the selective extraction of a particular analyte. This technology is gaining more and more attention due to the evolution on the way these materials are being synthetized, allowing to increase molecular recognition $[66,67]$. A particular example of this process includes molecularly imprinted microspheres (MIMs) used as sorbent [68]. In this work MIMs were prepared by aqueous suspension polymerization using 3-hydroxy-2-naphthoic acid and 1-methylpiperazine as mimic templates of the analytes and used as selective sorbents for IAA and IBA purification from banana samples. Because the template used for MIM synthesis was not one of the target analytes, the prepared MIMs, with a diameter distribution of $30-60$ 
$\mu \mathrm{m}$, were able to overcome the common problem of template leakage. Moreover, the MISPE procedure showed higher extraction efficiency and better selectivity than conventional C18-SPE [68]. An alternative variation of the MISPE method uses magnetic MIP (mag-MIP) beads as sorbent. Auxin-complementary mag-MIPs can be synthesized by microwave heating-induced polymerization of 4 -vinylpiridine and $\beta$-cyclodextrin and, after adsorption, can easily be collected with a magnetic bar, simplifying the isolation step [46,69]. Mag-MISPE has been applied to the extraction of IAA and IBA from wheat, pea and rice seeds [46,69] but IBA was never successfully extracted from any of the tested samples. This probably happened because IAA was used as a template to prepare the mag-MIPs and the selectivity obtained for IBA is not enough to extract the very low endogenous amounts normally present in plants $[20,28]$. Although MISPE can be advantageous in terms of increased specificity and faster purification than conventional SPE, the main disadvantage of this technique is the high amount of sample needed. At least in these initial reports, several grams of sample were used to produce a crude extract. It is likely that the required sample size will decrease with the development of the technology, but currently MISPE applied to auxin analysis still needs improvements.

Another adaptation of SPE based on magnetic properties of the sorbent was described by Liu et al. [70] for the quantification of IAA and other plant hormones from rice leaves. Instead of being packed into a cartridge, a magnetic sorbent made of $\mathrm{TiO}_{2} /$ magnetic hollow mesoporous silica spheres was dispersed into the sample by vortex, and could be easily separated from the sample by an external magnet. The adsorbed analyte was then derivatized in situ with 3bromoactonyltrimethylammonium bromide (BTA) in preparation for UPLC/MS/MS analysis. More recently, Cai et al. [71] used $\mathrm{Fe}_{3} \mathrm{O}_{4} @ \mathrm{TiO}_{2}$ magnetic nanoparticles, synthesized by liquidphase desorption (LPD), as sorbent for the purification of IAA and other plant hormones from rice seedlings. The purified analytes were further analyzed by UPLC/MS/MS. Because they are dispersed in solution and don't need to be packed into an SPE cartridge, magnetic adsorbents allow a faster sample preparation by dramatically increasing the contact surface area between sample and sorbent and by avoiding the column blocking step commonly used in conventional SPE [71]. However, despite the advantages named here and the potential of these techniques, several constraints impede their broad application in auxin analysis. A major disadvantage is the limited commercial availability of this type of sorbent, a lack which frequently implies in- 
house modification. Correct functionalization of magnetic nanoparticles may take several months and not all labs are equipped with the necessary tools for this kind of procedure. It may also lead to high variability between batches. Therefore, despite the future potential and elegance of these techniques, the inherent drawbacks that method development with magnetic particles may arise cannot be disregarded.

Adaptations of the classical LLE technique have also been described in the literature. Wu and $\mathrm{Hu}[24]$ introduced the hollow fiber-based liquid-liquid-liquid microextraction (HF-LLLME), where the analytes are transferred from an aqueous solution (donor phase) to another aqueous solution (acceptor phase), through an organic solvent (organic phase). The protocol is performed with inexpensive equipment and low solvent consumption; however, it was only applied to the quantification of IAA from coconut water samples. Although a good enrichment factor was obtained (215-fold), the applicability of the method to solid samples was not tested.

Microtechniques such as DLLME have been used in the extraction of auxins from the green algae Chlorella vulgaris [35]. This approach greatly reduced the extraction time $(<1 \mathrm{~min})$ and allowed good enrichment factors (10-fold for IAA and 60-fold for IBA). However, the same method could not be used for auxin quantification in the shrub Duranta repens due to "severe background interference" which represents a main disadvantage, as the method can't be applied to plant samples. Nevertheless, an analogous DLLME method was developed for the quantification of IAA and IBA from olive (Olea europaea) samples (Porfirio et al. 2015, unpublished). Actually this method was efficient in extracting auxins from two very different types of tissues (semi-hardwood cuttings and microcuttings) proving the reliability of DLLME as extraction/purification method for auxin analysis in plant samples.

\subsubsection{Purification by immunoaffinity columns}

Several authors have used immunoaffinity columns for the purification of plant extracts. Immunoaffinity purification is based on the highly selective antibody-antigen interaction and therefore significantly reduces common SPE problems such as co-extraction and matrix interferences [72]. Immunoaffinity columns are packed with sorbents that contain immobilized 
antibodies against a specific analyte, also called immunosorbents, allowing sample concentration [73]. Because low molecular mass compounds are unable to induce immune responses, the development of antibodies against these analytes includes their binding to a large carrier molecule, typically bovine serum albumin (BSA) [72], allowing protein recognition by the antibody. This was the case of the protocol developed by Pěnčik et al. [74], who generated IAA-BSA conjugates that were used to produce polyclonal antibodies in rabbit. In this work, samples (30 mg) of Helleborus niger were firstly extracted with phosphate buffer and prepurified by SPE. The resulting eluate was further purified in an immunoaffinity column containing immobilized polyspecific rabbit polyclonal antibodies against the IAA-BSA conjugate. Because IAA is attached to BSA through its carboxylic group, these antibodies are also able to interact with other indolic compounds such as indole-3-acetamide and indole-3-acetonitrile (IAA precursors) [74]. Although some cross-reaction can happen with IBA or IAA-Aspartate (IAAAsp), this issue is circumvented by methylation of the analytes with diazomethane before immunoaffinity purification. Indeed, this method allowed identification and quantification of several IAA conjugates including IAA-Glycine (IAA-Gly), IAA-Phenylalanine (IAA-Phe) and IAAValine (IAA-Val) in the $\mathrm{pg} \mathrm{g}^{-1} \mathrm{FW}$ range. Although IAA-Gly and IAA-Val had been previously described in crown gall cell cultures [75], this was the first report on these conjugates in higher plants.

Similar procedures were used by other authors to purify IAA and in some cases its conjugates from seaweed concentrates [76], roots of Ricinus communis infected with Agrobacterium tumefaciens [77] and tobacco BY-2 cells [55].

As previously mentioned, immunosorbents present major advantages in comparison with traditional sorbents. In fact, home-made immunosorbents can retain consistent analyte binding capabilities even after hundreds of utilizations over a period up to 1 year [72]. However, despite its superior behavior, immunoaffinity purification is most definitely not the main purification method used in auxin analysis, mainly because of the high costs associated with its operation, the difficulties in producing antibodies, or the high cost of commercially available antibodies, and the necessity of synthesizing analyte-protein conjugates capable of generating an immune 
response. Furthermore, the fact that reproducible immunosorbents can only be obtained with monoclonal antibodies [72] greatly increases the difficulty and cost of the entire procedure.

\subsubsection{Other purification methods}

Aside from the methods described above, less common purification strategies can also be found in the literature.

Schmelz et al. [22,78] used Super $Q$ filters and open-top capped vials to perform what they called vapor phase extraction (VPE) after a conventional sample pretreatment including tissue homogenization with an extraction solvent. In this protocol, pre-derivatized plant samples were heated at $200^{\circ} \mathrm{C}$, so that methylated IAA was volatilized and retained in the Super $\mathrm{Q}$ filters which were eluted for further $\mathrm{GC} / \mathrm{MS}-\mathrm{Cl}$ analysis.

Another example of a particular extraction procedure was described by Yin et al. [79], who used dual-cloud point extraction (dCPE) for quantification of IAA and IBA in acacia leaves, buds, and bean sprout. The procedure consists on the formation of a cloud point, mediated by a thermostatic bath, between an acidic aqueous solution and a surfactant resulting in the formation of two phases. The two phases are separated by centrifugation and, after increasing the viscosity of the surfactant phase with an ice bath, the aqueous phase is removed. Then the surfactant phase containing the analytes is mixed with an alkaline solution, into which the analytes will be extracted. A new cloud point is formed by incubation in a thermostatic bath and the resulting aqueous phase is collected after centrifugation.

Many references also use HPLC fractionation as a purification step before analysis $[50,55,80-$ 87], however, this procedure is very cumbersome and incompatible with high-throughput analysis, and protocols most recently developed focused in eliminating this step $[18,20]$.

Finally, among other plant hormones, IAA has been extracted from zucchini samples by the QuEChERS (acronym for quick, easy, cheap, effective, rugged and safe) methodology using $1 \%$ acetic acid in acetonitrile, anhydrous magnesium sulphate, sodium chloride, sodium citrate 
dehydrate and disodium citrate. However, the method was only able to extract IAA from one out of seven tested samples [88].

\subsubsection{Derivatization or labeling}

Derivatization refers to a group of modifications intended to make analytes more compatible with the detection method, ultimately increasing sensitivity and selectivity $[47,89]$. For instance, ionization in ESI-MS is frequently improved by derivatization [90-92], and IAA response in ESIMS/MS can increase up to 200-fold after methylation [93].

Several factors determine the choice of a derivatization procedure, including the analyte's chemical structure, separation method and type of detector. Incorporation of UV-absorbing or fluorescent groups is commonly used in LC and CE, and a large variety of reagents is available [89]. A decrease in polarity and increase in hydrophobicity, desirable both in GC and MEKC, is achieved by addition of alkyl, acyl or silyl groups [94]. In GC, an increase in volatility is also desirable for many compounds, including auxins, and this is often achieved through the addition of non-polar groups using silylation [34] and methylation [13] reactions. In fact, these are the derivatization procedures most commonly used in preparation of auxins for GC/MS analysis (Table S1), although examples of other reactions can also be found in the literature $[50,52,77]$.

Methylation is frequently accomplished using diazomethane, a reagent that specifically modifies the carboxylic group of auxins in a short reaction time [18]. Diazomethane is normally used in preparation of samples for GC/MS analysis, where, in the case of auxins, derivatization is mandatory, but it can also be applied to LC/MS analysis as a way of increasing the hydrophobicity of the analytes and improve separation $[44,51,95]$. It has also been applied before ELISA detection of IAA [84]. Besides diazomethane, other reagents have been used in derivatization reactions preceding LC/MS/MS analysis. One example is bromocholine, which contains a quaternary amine moiety and converts carboxyl groups in positively charged groups improving the detection of some plant hormones. Although auxins don't require this kind of modification, as they can be analyzed in positive ion mode, given their structure they are still modified in the reaction with bromocholine [53]. 
Like GC-FID and GC/MS, CE often requires derivatization. The reaction can occur in pre-, onor post-capillary modes, or even on-line (reviewed in [89]). Several examples of auxin modification can be found in the literature. In preparation for CE-electrochemiluminescent detection (CE-ECL), IAA has been derivatized through AEMP labeling with 2-(2-aminoethyl)-1methylpyrrolidine (AEMP) using N,N'-dicyclohexylcarbodiimide (DCC) and 3,4-dihydro-3hydroxy-4-oxo-1,2,3-benzotriazine (HOOBt) as coupling agents [79]. When using CE with laserinduced fluorescence detection (CE-LIF) several auxins were derivatized in situ with 6-Oxy(acetyl piperazine) fluorescein (APF) [96], a derivatizing reagent for carboxyl compounds that has also been applied to HPLC-FLD detection of auxins [97]. Recently, a new mass probe was developed for the detection of IAA and IBA by CE-ESI-TOF-MS. BTA contains a permanent positive charge that improves the ionization of acidic plant hormones, like auxins, allowing a better signal response in TOF-MS and multiple reaction monitoring (MRM) $[70,98]$. In fact, BTA has also been applied as in situ derivatization reagent to improve sensitivity in UPLC/MS/MS [70].

Derivatization can tremendously improve sensitivity, as demonstrated by Prinsen et al. [93]. This is especially important when dealing with low concentration analytes, such as auxins. While in the case of GC analysis derivatization is not optional, it can also improve auxins' response when using other techniques. Considering the low price of derivatizing reagents and the resulting analytical improvements, derivatization is an extremely important and advantageous step of sample preparation.

\subsection{Analysis}

The last step in the analytical process is, of course, analysis of the purified sample in its natural or derivatized form. Chromatographic techniques have long been the preferred methods for analysis of plant hormones. GC/MS and LC/MS provide the separation and sensitivity required for accurate quantification of compounds present in trace amounts in complex matrices, such as auxins [16]. Immunoassays also have been an important tool in plant hormone analysis, since early 1980's [99], and ELISA is still commonly applied to auxin quantification (Table S4). 
Nevertheless, other methods such as MEKC [100], pressurized capillary electrochromatography (pCEC) [101,102], CZE [103], CE [79,96,98,104] and surface plasmon resonance (SPR) [105] have also been applied.

\subsubsection{Separation and detection}

\subsubsection{Chromatographic methods}

Chromatography is the prevalent analytical technique for plant hormones, and because several reviews on this subject have been published in the past [14-16], only the most relevant approaches will be discussed here.

\subsubsection{GC and GC/MS}

GC/MS is the most classical method of auxin quantification. Although a few reports used GCECD for IAA quantification, compound identification was still performed by GC/MS in such cases [77]. More sensitive than LC/MS [41], GC/MS has been widely applied to auxin analysis, especially after $\left[{ }^{13} \mathrm{C}_{6}\right]$ IAA was chemically synthesized and proposed as internal standard for IAA quantification [106]. Although other standards, such as deuterated IAA $\left(\left[{ }^{2} \mathrm{H}_{2}\right] \mathrm{IAA}\right.$ and $\left.\left[{ }^{2} \mathrm{H}_{5}\right] \mathrm{IAA}\right)$ (Table S1) and methylated IAA (MeIAA) [49], are used sometimes, $\left[{ }^{13} \mathrm{C}_{6}\right]$ IAA offers several advantages over the deuterium labeled standards, namely, nonexchangeability of the isotope label, high isotopic enrichment, and chromatographic properties identical to that of the analyte [106].

Initially performed using SIM [107] or high-resolution SIM $[108,109]$, the sensitivity of this technique was highly improved with the development of multisector instruments that allow MRM. Hence, when accessible, this is currently the preferred mode of analysis when using GC/MS, allowing very good sensitivity and low detection limits (Table S1). One of the first examples of the use of GC/MS/MS on IAA quantification is the work of Müller et al. [21], who used a multiplex technique to quantify multiple acidic plant hormones in a single run. This method 
allowed them to generate whole-plant organ-distribution maps of IAA (among other plant hormones) in Arabidopsis thaliana. In the following years, other authors used GC/MS/MS to study auxin transport and synthesis in Arabidopsis and pea [33,34]. More recently, a highthroughput assay, which uses typically $2-10 \mathrm{mg} \mathrm{FW}$ of tissue, was developed for the quantification of IBA, IAA and IAA precursors in Arabidopsis and tomato [20].

\subsection{LC and LC/MS}

LC, coupled to various types of detectors, has also been broadly applied to auxin analysis (Table S2), and it is a more suitable technique than GC since no derivatization step is required. Given its sensitivity and selectivity, MS detection is most commonly used, and different mass analyzers are described in the literature: IT, quadrupole time-of-flight (QTOF), tandem quadrupole (qMS/MS), and triple quadrupole linear ion trap (Q-Trap) (see Table S2). Currently, IAA and IBA can be separated from other auxins within 7 min by LC/ESI-ITMS [110]. With the development of LC/MS instruments, MRM mode became a reality and the technique surpassed GC/MS due to its simplicity. Currently, it is the most commonly used method of auxin quantification [16] (Table S2).

Recently LC/MRM-MS was used to analyze the Arabidopsis IAA metabolome from amounts of tissue as small as $20 \mathrm{mg}$. In the same protocol, most IAA precursors and degradation products were analyzed simultaneously, demonstrating the analytical power of this technique. However, in all studied tissues, IBA levels were below the detection limit of the method [42]. Sensitivity and detection limits can be highly improved by the use of nanoflow-LC/MRM-MS. Izumi et al. [111] reported detection limits in the fmol range and a 5-10 fold increase in sensitivity when using nanoflow-LC/ESI-IT-MS/MS with MRM for plant hormone profiling.

Some authors have also performed two-dimensional HPLC (2D-HPLC) for auxin quantification. Dobrev et al. [112] firstly described a "heart-cutting" 2D-HPLC method to separate and purify IAA and abscisic acid (ABA) from several plant species. In the first dimension, a silicacyanopropyl column was used and the elution was performed in reverse-phase mode at a low concentration of organic solvent, allowing a close elution of the two analytes. This was 
beneficial for the separation in the second dimension, which was done in a hydrophobic $\mathrm{C} 18$ column, because it concentrated IAA and ABA in narrow peaks. The full injection-to-injection cycle was smaller than 30 min and the analytes were detected by DAD and fluorescence (FLD) detectors connected in series. The method was subsequently used by other authors $[113,114]$. comprehensive (LC x LC) 2D-HPLC/DAD method for metabolomics studies. The speed of the second dimension separation was improved by using an ultra-fast and high temperature gradient elution, which reduced cycle time. For that purpose, a high-pressure mixing configuration was used to generate each second dimension gradient, instead of two separate binary pump systems. This design eliminated the differential in retention time between sequential separations, allowing a reduction in dwell volume higher than an order of magnitude. The two columns used (Discovery HS-F5 and ZirChrom-CARB, first and second dimension, respectively) allowed a high degree of orthogonality and thus the method was used to separate 26 IAA derivatives from maize samples in a single injection cycle, in a practical analysis time of $25 \mathrm{~min}$.

2D-LC has the potential to be an extremely powerful separation technique mainly due to its exceptional resolving power compared to conventional 1D-LC methods (see [116] for a thorough review). In theory, it has a very broad application range as it allows performing separations using a large combination of LC modes (SEC, RP, IEC, etc.), although in practice the combination of certain modes is very difficult, if not impossible [117]. Despite the tremendous potential of 2D-LC, several disadvantages prevent its widespread use. The main drawback is still the very long timescale of comprehensive analysis (several hours). Unlike 2DGC, where high speed separation is easier to implement, high speed separation in LC is more difficult because of pressure and instrumentation limitations (discussed in detail in [116]). As mentioned above, this problem was partially addressed by Stoll et al. [115], who were able to reduce the overall separation time to about $30 \mathrm{~min}$ by increasing the temperature and linear velocity of the second dimension column. An alternative to high temperatures in speeding up the second dimension analysis can be the use of monolithic columns, which can accommodate high flow rates without loss of resolution [117]. 
Furthermore, the number of parameters that need to be chosen, combined and optimized 450 (column, flow rates, mobile phases, gradients and temperatures) for a 2D-LC method is considerably higher than for a 1D-LC method [116], which significantly increases the complexity of the technique. Combining 2D-LC separation with an MS detector is also a challenge considering that the flow rate has to be significantly reduced in order to be compatible with ESI [116]. The speed of the detector is also a problem, especially in the case of MS detectors, which can be slower than the LC separation [116].

Another major drawback of 2D-LC is data analysis. The amount of data resulting from a comprehensive 2D-LC analysis can be overwhelming, especially when using an MS or PDA detector, and currently there are no commercially available softwares that allow efficient and semi-automated analysis of 2D-LC data [117], although this reality may change in the near future.

461

Unlike 2D-GC, which was invented over two decades ago $[118,119]$, has been continuously developed ever since, and is currently automated and commercially available, 2D-LC is still far from routine. To this date, $2 \mathrm{D}-\mathrm{LC}$ remains still a promising technique.

Nevertheless, LC/MS accuracy and sensitivity is increasing even in 1D separations. Recently, high-resolution and accurate mass instruments have been used for the identification of a wide range of indolic compounds from crude plant extracts. A minimalistic sample purification protocol involving only centrifugation and dilution of the organic extract followed by quadrupole ion cyclotron resonance Fourier transform MS (Q ICR FT-MS) analysis allowed the identification of multiple indolic compounds, including free IAA, IAA amide-conjugates, tryptophan conjugates and other tryptophan derivatives from soybean, tomato and Ginkgo biloba $[26,120]$. Additionally, separation and quantification of four isomers of auxin-myo-inositol conjugates (IAA-Inos), as well as IAA and MeIAA, from Zea mays and Arabidopsis thaliana was also reported using QTOFMS [121]. 


\subsection{Electrokinetic methods}

477 Although chromatographic methods are the prevalent analytical strategy to study plant 478 hormones, separation is hampered by the complex sample matrix and the high cost of analysis 479 resulting from the need for isotopically-labeled internal standards [96]. CE has a higher 480 separation power than LC and GC, which are based on the interaction between analytes and 481 the stationary phase, because analytes are separated according to their electrokinetic 482 properties (i.e., mass and charge) [122]. Thus, separations with several hundred thousand 483 theoretical plates can be achieved with CE [123]. Furthermore, CE displays several advantages 484 over chromatography such as low sample (sub-nL) and reagent (sub- $\mu \mathrm{L})$ consumption, short 485 separation time and low instrumentation cost $[123,124]$. Several examples of the application of 486 CE to auxin quantification can be found in the literature (Table S3).

487 A method based on CE-LIF was developed for auxin quantification from crude banana extracts, using APF as derivatizing reagent [96]. CZE was firstly tested as separation mode, but all analytes were flushed together using these conditions. Therefore MEKC was chosen as separation mode and all parameters ( $\mathrm{pH}, \mathrm{SDS}$, ethanol concentration, water content) were optimized. Under optimized conditions, and without any sample clean-up, IAA and IBA were separated from other plant hormones and quantified within 20 min, with detection limits in the $\mathrm{nM}\left(\mu \mathrm{g} \mathrm{mL} \mathrm{L}^{-1}\right)$ range [96].

In other cases, CE-electrochemiluminescence (ECL) was used to analyze IAA and IBA from acacia tender leaves, buds, and bean sprout [79]. ECL detection is based on the formation of photons resulting from the decay of species that easily form excited states at the surface of electrodes, via an applied voltage. Among ECL systems, tris (2,2'-bipyridyl)ruthenium(II) $\left(\mathrm{Ru}(\mathrm{bpy})_{3}{ }^{2+}\right)$ is one of the most commonly used, especially when in combination with CE [123]. Oxidation of $\mathrm{Ru}(\mathrm{bpy})_{3}{ }^{2+}$ by analytes containing tertiary amines generates an excited-state $\mathrm{Ru}(\mathrm{bpy}){ }_{3}{ }^{2+{ }^{*}}$, whose decay to the steady state leads to the release of a photon. The amount of light energy released is therefore proportional to the analyte's concentration. The method is a powerful analytical tool with high sensitivity and wide linear ranges [123], but its application requires the presence of tertiary amine groups in the analytes. Analytes lacking a tertiary amine group in their structure, such as auxins, can be derivatized with AEMP in order to increase 
detection sensitivity [79]. Although this is a feasible solution, it also introduces an extra step in sample preparation. Good detection limits were obtained (nM), and the method was validated by HPLC-UV detection. $\mathrm{Ru}(\mathrm{bpy})_{3}{ }^{2+}-\mathrm{KMnO}_{4} \mathrm{ECL}$ had been used previously for the detection of IAA and IBA in mung bean sprouts [125]. In this work, $\mathrm{Ru}(\mathrm{bpy}){ }_{3}{ }^{2+}$ was immobilized on an anionexchange resin and the $\mathrm{ECL}$ reaction happened by contact with a diluted acidic $\mathrm{KMnO}_{4}$ solution. This design reduces reagent consumption and does not require a mixing chamber or a pump. Furthermore, the reagent-containing resin could be used for at least six months as the relative ECL intensity only decreased $3 \%$ during that period.

Hybrid techniques like CEC and PCEC combine the efficiency of CE and the selectivity of HPLC, overcoming the disadvantages of CE [122]. In the case of pCEC, both a pressurized flow and the EOF push the mobile phase through the capillary, thus solving the problems associated with column drying-out and bubble formation [101]. Wang et al. [101] used pCEC to quantify IAA extracted from corn. Separation was carried out in a monolithic silica-ODS column, and detection was accomplished in a UV-Vis detector. Although the authors pointed out some disadvantages of $\mathrm{PCEC}$, such as low concentration sensitivity associated with low sample volume and limited optical path length for UV-Vis detection, pCEC provided a better separation than LC. Yin and Liu [122] developed a method for the preparation of polydopamine-coated open-tubular capillary columns to be used in the detection of IAA and IBA. The capillary is filled with an aqueous solution of dopamine, and polydopamine is formed in the inner wall of the capillary through oxygen-derived oxidation of the dopamine solution, forming a permanent coating. The use of repetitive coatings allowed the formation of a layer with $200 \mathrm{~nm}$ thickness, providing a column with controllable EOF (the coating inhibits EOF, possibly by masking silanol groups in the inner wall of the capillary). The developed coating was stable under both acidic and alkaline conditions, resistant to the presence of methanol in the sample, and it can be stored for up to 2 months. Even though the coating was developed to separate IAA and IBA, a decreased interaction of IBA and the coating was observed, likely due to its longer chain. Nevertheless, both auxin standards were separated within $11 \mathrm{~min}$ in the single layer polydopamine-coated capillary, which showed improved resolution in comparison to the bare capillary. Although the method was successfully applied to the determination of IAA in culture 
media of IAA-producing bacteria (Arthrobacter sp., Bacillus sp. and Enterobacter sp.), its applicability in plant samples was not evaluated.

\subsection{Immunoassays}

Immunoassays such as RIA and ELISA have long been applied to auxin quantification $[99,126]$. They are based on highly specific antibody-antigen interactions where the analyte is an auxin conjugate that can be recognized by the antibody. Although ELISA is much less sensitive than LC/MS for the detection of plant hormones [127], and presents several obstacles such as complex sample preparation and cross-reactivity [16], its high selectivity and ease of operation make it a valuable tool for auxin analysis. Furthermore, anti-auxin monoclonal antibodies and full ELISA kits are commercially available, which is not true for other analytical techniques. ELISA has been used for IAA quantification using IAA-ovalbumin or IAA-BSA conjugates $[77,128,129]$.

Aside from ELISA assays, biosensors are gaining popularity due to their unique properties. Different kinds of biosensors have been adapted to plant hormone determination [130] and, particularly, immunosensors provide high specificity and sensitivity due to the use of antibodies or antigens as the sensing element [16].

\subsection{Immunosensors and other biosensors}

Biosensors are analytical devices that combine a biological component with a physicochemical detector, and convert a biological response into a signal that can be captured and interrogated [130]. As stated by Sadanandom and Napier [130], the ideal biosensor is selective, sensitive, gives a calibrated dose-response curve over physiologically relevant concentrations of analyte, gives a spatially resolved reading in vivo, and is not invasive.

A specific type of biosensor is an immunosensor, where the immunochemical reaction is coupled to a transducer and converted into an electrical signal (reviewed in [131]). 
Immunosensors can be classified based on the type of detector: electrochemical, optical and piezoelectric [131].

An immunosensor with a piezoelectric detector was designed for IAA detection [132]. A piezoelectric detector consists of a quartz crystal microbalance (QCM) which detects mass differences between the analyte-bound an unbound states of the biosensor. Because IAA is too small to produce a sufficient mass difference, an IAA-BSA conjugate with higher molecular weight was synthesized and used as antigen (analyte), increasing the sensitivity of the assay. Anti-[IAA-BSA] antibodies were purified from white rabbits and immobilized on the golden surface of the quartz crystal. This configuration allowed creating a QCM immunosensor capable of detecting IAA in a linear range of $0.5 \mathrm{ng} \mathrm{mL}^{-1}-5 \mu \mathrm{g} \mathrm{m}^{-1}$. The capacity of the immunosensor was evaluated by determining IAA in solution at different concentrations, in the range $1 \mathrm{ng} \mathrm{mL}^{-1}$

$571-1 \mu \mathrm{g} \mathrm{mL}^{-1}$, and the calculated recoveries varied from 96 to $116 \%$. However, although a 572 functional immunosensor was developed, at the time of the study regeneration of the biosensor 573 was an unsolved problem, which creates a great disadvantage. This problem was addressed in 574 later work by the same authors who developed a renewable amperometric immunosensor for 575 IAA detection [133]. This immunosensor consists of a sol-gel-alginate-carbon composite electrode (SACE), produced from the sol-gel precursor tetramethoxysilane (TMOS), alginate

577 and graphite powder, and contains anti-IAA antibodies on its surface. The detection was based on the enzyme-linked competitive immunoreaction between IAA in the sample and IAA labeled 579 with horseradish peroxidase (IAA-HRP) on the SACE surface. The enzymatic activity of HRP 580 bound to anti-IAA antibodies is measured by amperometric detection using $\mathrm{H}_{2} \mathrm{O}_{2}$ and $3,3^{\prime}, 5,5^{\prime}-$ 581 tetramethylbenzidine (TMB) as substrates. This biosensor was capable of detecting IAA in the 582 range $5-500 \mu \mathrm{gL}^{-1}$ and was applied to the analysis of hybrid rice grain samples. After each 583 immunoassay, the sensor was regenerated by immersing the SACE in saline solution at $\mathrm{pH} 12$ 584 in order to rinse out the antibody immobilized on the SACE surface. The method was validated 585 by analyzing the rice samples by HPLC.

586 Other types of biosensors were further developed for IAA detection. Mancuso et al. [134] 587 described a non-invasive carbon-nanotube modified and self-referencing microelectrode for the 588 study of auxin fluxes in root apexes. It is desirable that the microelectrode can detect IAA levels 
at precise distances from the tissues with good spatial resolution. Carbon nanotubes have high electrical conductivity, chemical stability and mechanical strength [134], solving some of the problems observed in previously developed electrodes [135]. Modifying the electrode surface with multiwalled carbon nanotubes increases the surface area available for electron transfer and enhances catalysis [136]. In fact, the authors showed the enhancing effect of multiwalled nanotubes on the oxidation peak current of IAA in comparison with a bare platinum electrode, further confirming the results of Wu et al. [137], who developed a similar but invasive electrode. The microelectrode created by Mancuso et al. [134] was used to monitor IAA fluxes in growing roots of maize, Arabidopsis and walnut. The method was validated by analyzing the samples by HPLC, and in both methods the amounts found in samples were in the $\mathrm{ng} \mathrm{g}^{-1}$ range.

Nevertheless, this method displayed some disadvantages such as lower than desired temporal resolution and signal-to-noise ratio, as well as the need for exogenous IAA addition. Further improvements of this approach were already reported by McLamore et al. [136], who optimized a non-invasive self-referencing electrochemical microsensor for the measurement of endogenous IAA fluxes in maize roots. The microsensor included platinum black and carbon nanotube (CNT) surface modifications and can be used for real-time transport monitoring in surface tissues. Furthermore, the method can be performed simultaneously with live imaging techniques.

Zhou et al. [138] developed an electrochemical immunosensor based on gold nanoparticles (AuNPs) functionalized with HRP-labeled immunoglobulins (HRP-lgGs) and rat monoclonal antibodies against IAA (anti-IAA). A glassy carbon electrode was coated with graphene for an increased electrode surface and to facilitate electron transfer. The AuNPs were deposited on the electrode surface to allow IAA recognition. The HRP-labeling was used as a signal amplification tool to increase the sensitivity of the immunosensor, while IAA recognition and capture was performed by the monoclonal anti-IAA antibodies attached to the AuNPs-HRP-IGs.

614 Electrochemical measurements were performed by differential pulse voltammetry (DPV) using $\mathrm{Fe}(\mathrm{CN})_{6}{ }^{3-} /^{4-}$ as redox probe, and IAA was indirectly measured by the variation of oxidation current response of $\mathrm{Fe}(\mathrm{CN})_{6}{ }^{3-} / \mu^{4-}$. The determined LOD was comparable with other techniques

617 (CE, chemiluminescence), and the method was applied to IAA quantification in mung bean 
sprouts $\left(12-32 \mathrm{ng} \mathrm{g}^{-1}\right)$. A very similar immunosensor was described in the same year [139].

619 The IAA detection mechanism is the same, however in this case 4-aminophenylboronic acid (4-

620 APBA) was used instead of graphene as coating agent for the electrode. Furthermore, in this

621 case the HRP-IgGs were attached to $\mathrm{Fe}_{3} \mathrm{O}_{4}-\mathrm{COOH}$ magnetic nanoparticles while the anti-IAA

622 antibodies were attached to the AuNPs, allowing double signal amplification. Also in this case

623 the results from IAA quantification in seeds (wheat, corn, mung bean, soy bean, millet and

624 brown rice) were comparable with results obtained by CE [139]. The LODs of both

625 immunosensors are comparable (nM range).

626 Another example of the use of graphene in electrodes is the work of Sun et al. [140] who 627 reported a photoelectrochemical (PEC) immunosensor using 3-mercaptopropionic acid 628 stabilized CdS/reduced graphene oxide (MPA-CdS/RGO) nanocomposites for IAA detection. In 629 this case graphene was chosen for its properties as electron-transfer matrix. PEC sensing is a 630 promising technique that allows high sensitivity and high-throughput while using inexpensive 631 devices, although to the best of our knowledge this is the only report describing the use of the 632 technique. The immunosensor was successfully applied to IAA quantification from wheat, corn 633 and bean seeds, with results comparable to those obtained using CE.

634 Yang et al. [141] developed an amperometric sensor based on a $\mathrm{CeCl}_{3}$-DHP film modified gold 635 electrode for IAA determination. In comparison with the bare and DHP modified gold electrodes, 636 this sensor greatly increased the linear response of detection while decreasing the noise of the 637 amperometric response. Mung bean sprout leaves were analyzed by this method, and results 638 were comparable with HPLC analysis. Previously, a carbon paste electrode had been developed for IAA quantification using square wave voltammetric determination based on surfactant effects [142]. This method presented very high sensitivity and low detection limits (20 nM) and was successfully applied to gladiola and phoenix tree leaves.

642 Finally, SPR was also applied to IAA monitoring in plant tissues [105]. SPR is a surface643 sensitive technique based on the measurement of changes in refractive index (RI), which allows 644 performing real-time and label-free analyte detection in complex matrices even without sample 645 pre-treatment. Sensitivity can be highly improved by coating the sensor chip surface with a thin 646 film of MIPs, which will selectively recognize a template molecule. In the work of Wei et al. [105], 
high selectivity was achieved by adsorbing a molecularly imprinted monolayer (MIM) on the SPR sensor chip surface containing preadsorbed IAA. The MIM consisted of a 2D monolayer of alkanethiol self-assembled around the template (IAA) pre-adsorbed on the surface of the goldcoated sensor chip. Selectivity was evaluated by applying the MIM-coated chip to the detection of IBA, and a much lower response was observed in this case. Moreover, an IBA-imprinted MIM was prepared which further showed the high selectivity of MIMs. The method was applied to different samples with good recoveries $(95-98 \%)$ and very good detection limits $(0.20-0.32$ $\mathrm{pM})$. The biggest disadvantage of this approach is the high cost of the SPR sensor chip, and its further functionalization with MIMs.

\subsection{Other detection methods}

A fluorimetric assay based on the reaction between IAA and acetic anhydride in the presence of perchloric acid as catalyst was used to quantify IAA in mung bean cuttings $[143,144]$. The method is highly specific as IBA didn't form detectable amounts of fluorescent derivatives, which can also be a disadvantage as no other auxins can be detected.

A colorimetric method for the detection of both IAA and IBA was described by Guo et al. [145], based on the reaction between auxins and Ehrlich reagent ( $p$-(dimethylamino)benzaldehyde (PDAB)) under acidic conditions. PDAB reacts with indolic compounds, and IAA and IBA respond differently to reaction temperature and incubation time. While at $25^{\circ} \mathrm{C}$ IBA's reaction with PDAB generates a blue compound, IAA barely reacts with PDAB at this temperature during the first 60 min, allowing selective determination of IBA absorbance. At $70^{\circ} \mathrm{C}$, both auxins react intensively with PDAB, however forming products with different colors (IBA - blue; IAA - pink). Therefore, IAA concentration can be determined as the difference between the total absorbance and IBA absorbance. The specificity of the method was tested by determining IAA and IBA concentration in the presence of other auxins (phenylacetic acid (PAA), naphtaleneacetic acid (NAA) and indole-3-carboxylic acid (ICA)) and tryptophan (Trp). Although no interference from PAA, NAA and Trp was observed, ICA is a powerful interferent, and IAA and IBA determination cannot be performed in its presence. The method had linear ranges of $0.28-56 \mu \mathrm{M}(\mathrm{IAA})$ and 
$0.84-42 \mu \mathrm{M}$ (IBA) with detection limits of $0.10 \mu \mathrm{M}$ (IAA) and $0.28 \mu \mathrm{M}$ (IBA). It was applied to the analysis of bean sprouts and results were comparable to results obtained by CE-ECL.

677 Although a wide variety of methods are available for auxin isolation and analysis, conventional 678 (i.e. chromatographic) methods are still the predominant techniques used in this field, because 679 they are well established within the laboratory setting as well as in literature. Techniques such 680 as biosensors and immuno-based methods provide novel alternatives to the field of study, but 681 the overall investment needed to implement and incorporate them into existing laboratories may 682 be still prohibitive for many laboratories. Furthermore, issues such as high operation cost, 683 limited commercial availability, low signal-to-noise ratios, standardization and low or nonselective specificity are some of the drawbacks to consider $[72,146,147]$. Therefore, although worth noting for this review, these alternative approaches still remain difficult to implement in a broad scale.

687

\section{Conclusions}

689 Analytical methods for auxin analysis have greatly evolved since they were first described 690 (reviewed in [41]) and more accurate, sensitive, precise and high-throughput methods are available nowadays. Although sample preparation remains the bottleneck of the analytical process, currently there are several approaches that can process large numbers of samples per day. A broad range of sample preparation methods is available, including the classical SPE and LLE and their respective variations (SPME, MISPE, DLLME, HF-LLLME), as well as alternative techniques such as VPE, QuEChERS, dCPE and immunoextraction. Many options are also available for sample analysis including GC, GC/MS, GC/MS/MS, LC, LC/MS, LC/MS/MS, CE,

697 CE/MS, immunoassays and other reported methods. Among these, LC/MS and especially LC/MS/MS are the most advantageous methods considering their excellent selectivity, superior sensitivity, high-throughput and high accuracy. It is also desirable that auxin analysis, as well as the simultaneous analysis of all plant hormones (hormone profiling, or hormonome [53]), becomes a routine practice. The development of 2D-LC has brought us closer to this goal, and 
can become a reality. Moreover, the comprehensive analysis of a plant's metabolome can also help understand, at the molecular level, the plant's response to different conditions such as stress, mutations, and hormone treatments. However, the chemical diversity of plant hormones poses a great challenge to the development of simple, efficient, fast and universal methods [16], a problem exponentially bigger in the case of metabolomics analysis. Another obstacle to this ideal analytical method is the current lack of automation for many sample preparation techniques, a problem that is being addressed in some cases [18,20], but still lacks a universal solution.

As stated by $\mathrm{Du}$ et al. [16], the development of techniques that allow highly sensitive, noninvasive, in vivo, in situ and real-time detection of auxins and other plant hormones is still an ambition. For example, while MS imaging (MSI) has been used with great success to map the spatial distribution of small metabolites in animal tissues [148], its application to the study of plant tissues has only recently begun $[149,150]$. Although MSI only allows a qualitative analysis, it will definitely be a valuable tool that can be used in combination with other techniques such as in vivo SPME [151,152], SPR-based biosensors [153,154] and atomic and molecular MS [155]. All these techniques can be powerful tools in the future study of the complex metabolic pathways associated with plant hormones.

\section{Acknowledgements}

Authors acknowledge funding from the Portuguese Foundation for Science and Technology (FCT), through the projects PTDC/AGR - AM/103377/2008 and PEst-C/AGR/UI0115/2011, through the Programa Operacional Regional do Alentejo (InAlentejo) Operation ALENT-070262-FEDER-001871 and through the Doctoral grant SFRH/BD/80513/2011. Authors also acknowledge funding from FEDER funds through the Competitiveness Factors Operational Program (COMPETE) and from the American Department of Energy (DOE) grant number DEFG02-93ER20097 for the Center for Plant and Microbial Complex Carbohydrates at the CCRC. The first author would also like to acknowledge Parastoo Azadi at the Complex Carbohydrate Research Center (CCRC) for gracious support in her research while in the United States. 


\section{References}

[1] A. Santner, M. Estelle, Recent advances and emerging trends in plant hormone signalling, Nature. 459 (2009) 1071-1078.

[2] L. Taiz, E. Zeiger, Plant physiology, 5th ed., Sinauer Associates, Sunderland, MA, USA, 2010.

[3] C. Darwin, F. Darwin, The power of movement in plants, John Murray, 1880.

[4] F.W. Went, Auxin, the plant growth-hormone, Bot. Rev. 1 (1935) 162-182.

[5] J.E. Preece, A century of progress with vegetative plant propagation, HortScience. 38 (2003) 1015-1025.

[6] A. Fabbri, G. Bartolini, M. Lambardi, S. Kailis, Olive propagation manual, CSIRO, 2004.

[7] J. Ludwig-Müller, A. Vertocnik, C.D. Town, Analysis of indole-3-butyric acid-induced adventitious root formation on Arabidopsis stem segments, J. Exp. Bot. 56 (2005) 2095-2105.

[8] C.M. Fogaça, A.G. Fett-Neto, Role of auxin and its modulators in the adventitious rooting of Eucalyptus species differing in recalcitrance, Plant Growth Regul. 45 (2005) 1-10.

[9] G. Osterc, F. Štampar, Differences in endo/exogenous auxin profile in cuttings of different physiological ages, J. Plant Physiol. 168 (2011) 2088-2092.

[10] P. Overvoorde, H. Fukaki, T. Beeckman, Auxin control of root development, Cold Spring Harb. Perspect. Biol. 2 (2010).

[11] K. Wei, L. Wang, H. Cheng, C. Zhang, C. Ma, L. Zhang, W. Gong, L. Wu, Identification of genes involved in indole-3-butyric acid-induced adventitious root formation in nodal cuttings of Camellia sinensis (L.) by suppression subtractive hybridization, Gene. (2013).

[12] T.I. Pop, D. Pamfil, C. Bellini, Auxin control in the formation of adventitious roots, Not. Bot. Horti Agrobotanici Cluj-Napoca. 39 (2011) 307-316.

[13] L.C. Strader, A.H. Culler, J.D. Cohen, B. Bartel, Conversion of endogenous indole-3-butyric acid to indole-3-acetic acid drives cell expansion in Arabidopsis seedlings, Plant Physiol. 153 (2010) 1577-1586.

[14] Y. Bai, F. Du, H. Liu, Determination strategies of phytohormones: recent advances, Anal.Methods. 2 (2010) 1867-1873.

[15] J. Fu, X. Sun, J. Wang, J. Chu, C. Yan, Progress in quantitative analysis of plant hormones, Chinese Sci. Bull. 56 (2011) 355-366.

[16] F. Du, G. Ruan, H. Liu, Analytical methods for tracing plant hormones, Anal. Bioanal. Chem. 403 (2012) 55-74.

[17] X. Pan, X. Wang, Profiling of plant hormones by mass spectrometry, J. Chromatogr. B. 877 (2009) 2806-2813.

[18] L.S. Barkawi, Y.-Y. Tam, J.A. Tillman, J. Normanly, J.D. Cohen, A high-throughput method for the quantitative analysis of auxins, Nat. Protoc. 5 (2010) 1609-1618.

[19] M.M. Koek, R.H. Jellema, J. van der Greef, A.C. Tas, T. Hankemeier, Quantitative metabolomics based on gas chromatography mass spectrometry: status and perspectives, Metabolomics. 7 (2011) 307-328.

[20] X. Liu, A.D. Hegeman, G. Gardner, J.D. Cohen, Protocol: high-throughput and quantitative assays of auxin and auxin precursors from minute tissue samples, Plant Methods. 8 (2012) 1-17.

[21] A. Müller, P. Düchting, E.W. Weiler, A multiplex GC-MS/MS technique for the sensitive and quantitative single-run analysis of acidic phytohormones and related compounds, and its application to Arabidopsis thaliana, Planta. 216 (2002) 44-56.

[22] E.A. Schmelz, J. Engelberth, J.H. Tumlinson, A. Block, H.T. Alborn, The use of vapor phase extraction in metabolic profiling of phytohormones and other metabolites, Plant J. 39 (2004) 790- 
808.

[23] Z. Ma, L. Ge, A.S. Lee, J.W.H. Yong, S.N. Tan, E.S. Ong, Simultaneous analysis of different classes of phytohormones in coconut (Cocos nucifera L.) water using high-performance liquid chromatography and liquid chromatography-tandem mass spectrometry after solid-phase extraction, Anal. Chim. Acta. 610 (2008) 274-281.

[24] Y. Wu, B. Hu, Simultaneous determination of several phytohormones in natural coconut juice by hollow fiber-based liquid-liquid-liquid microextraction-high performance liquid chromatography, J. Chromatogr. A. 1216 (2009) 7657-7663.

[25] J. Fu, J. Chu, X. Sun, J. Wang, C. Yan, Simple, Rapid, and Simultaneous Assay of Multiple Carboxyl Containing Phytohormones in Wounded Tomatoes by UPLC-MS/MS Using Single SPE Purification and Isotope Dilution, Anal. Sci. 28 (2012) 1081-1087.

[26] P. Yu, A.D. Hegeman, J.D. Cohen, A facile means for the identification of indolic compounds from plant tissues, Plant J. 79 (2014) 1065-1075.

[27] Y. Chen, Z. Guo, X. Wang, C. Qiu, Sample preparation, J. Chromatogr. A. 1184 (2008) 191-219.

[28] X. Pan, R. Welti, X. Wang, Quantitative analysis of major plant hormones in crude plant extracts by high-performance liquid chromatography-mass spectrometry, Nat. Protoc. 5 (2010) 986-992.

[29] M. Müller, S. Munné-Bosch, Rapid and sensitive hormonal profiling of complex plant samples by liquid chromatography coupled to electrospray ionization tandem mass spectrometry, Plant Methods. 7 (2011) 37.

[30] I. Machakova, E. Zazimalova, E.F. George, Plant growth regulators I: Introduction; auxins, their analogues and inhibitors, in: George, Edwin F and Hall, Michael A and De Klerk, Geert-Jan (Ed.), Plant Propagation by Tissue Culture, Springer, Dordrecht, The Netherlands, 2008: pp. 175-204.

[31] Y. Kim, Y. Oh, W. Park, HPLC-based quantification of indole-3-acetic acid in the primary root tip of maize, J. Nano. Bio. Tech. 3 (2006) 40-45.

[32] I. Nakurte, A. Keisa, N. Rostoks, Development and Validation of a Reversed-Phase Liquid Chromatography Method for the Simultaneous Determination of Indole-3-Acetic Acid, Indole-3Pyruvic Acid, and Abscisic Acid in Barley (Hordeum vulgare L.), J. Anal. Methods Chem. 2012 (2012) 6 pages. doi:10.1155/2012/103575.

[33] S.E. Jones, J.S. DeMeo, N.W. Davies, S.E. Noonan, J.J. Ross, Stems of the Arabidopsis pin1-1 mutant are not deficient in free indole-3-acetic acid, Planta. 222 (2005) 530-534.

[34] L.J. Quittenden, N.W. Davies, J.A. Smith, P.P. Molesworth, N.D. Tivendale, J.J. Ross, Auxin biosynthesis in pea: characterization of the tryptamine pathway, Plant Physiol. 151 (2009) 11301138.

[35] Q. Lu, L. Chen, M. Lu, G. Chen, L. Zhang, Extraction and analysis of auxins in plants using dispersive liquid- liquid microextraction followed by high-performance liquid chromatography with fluorescence detection, J. Agr. Food Chem. 58 (2010) 2763-2770.

[36] F. Matsuda, H. Miyazawa, K. Wakasa, H. Miyagawa, Quantification of indole-3-acetic acid and amino acid conjugates in rice by liquid chromatography-electrospray ionization-tandem mass spectrometry, Biosci. Biotech. Biochem. 69 (2005) 778-783.

[37] H.-T. Liu, Y.-F. Li, T.-G. Luan, C.-Y. Lan, W.-S. Shu, Simultaneous determination of phytohormones in plant extracts using SPME and HPLC, Chromatographia. 66 (2007) 515-520.

[38] X. Liu, L. Barkawi, G. Gardner, J.D. Cohen, Transport of indole-3-butyric acid and indole-3-acetic acid in Arabidopsis hypocotyls using stable isotope labeling, Plant Physiol. 158 (2012) 1988-2000.

[39] J.C. Gonçalves, G. Diogo, M.T. Coelho, N. Vidal, S. Amâncio, Quantitation of endogenous levels of IAA, IAAsp and IBA in micro-propagated shoots of hybrid chestnut pre-treated with IBA, In Vitro Cell. Dev. Biol.-Plant. 44 (2008) 412-418.

[40] G. Absalan, M. Akhond, L. Sheikhian, Extraction and high performance liquid chromatographic determination of 3-indole butyric acid in pea plants by using imidazolium-based ionic liquids as 
extractant, Talanta. 77 (2008) 407-411.

[41] N.D. Tivendale, J.D. Cohen, Analytical History of Auxin, J. Plant Growth Regul. (2015) DOI 10.1007/s00344-015-9519-4.

[42] O. Novák, E. Hényková, I. Sairanen, M. Kowalczyk, T. Pospíšil, K. Ljung, Tissue-specific profiling of the Arabidopsis thaliana auxin metabolome, Plant J. 72 (2012) 523-536.

[43] Y. Liang, X. Zhu, T. Wu, M. Zhao, H. Liu, Rapid and sensitive detection of auxins and flavonoids in plant samples by high-performance liquid chromatography coupled with tandem mass spectrometry, J. Sep. Sci. 35 (2012) 2559-2566.

[44] M. Kowalczyk, G. Sandberg, Quantitative analysis of indole-3-acetic acid metabolites in Arabidopsis, Plant Physiol. 127 (2001) 1845-1853.

[45] A. Liazid, M. Palma, J. Brigui, C.G. Barroso, Investigation on phenolic compounds stability during microwave-assisted extraction, J. Chromatogr. A. 1140 (2007) 29-34.

[46] Y. Hu, Y. Li, Y. Zhang, G. Li, Y. Chen, Development of sample preparation method for auxin analysis in plants by vacuum microwave-assisted extraction combined with molecularly imprinted clean-up procedure, Anal. Bioanal. Chem. 399 (2011) 3367-3374.

[47] L. Ramos, Critical overview of selected contemporary sample preparation techniques, J. Chromatogr. A. 1221 (2012) 84-98.

[48] K. Cui, Y. Lin, X. Zhou, S. Li, H. Liu, F. Zeng, Comparison of sample pretreatment methods for the determination of multiple phytohormones in plant samples by liquid chromatography-electrospray ionization-tandem mass spectrometry, Microchem. J. 121 (2015) 25-31.

[49] J. Rolčík, J. Řečinská, P. Barták, M. Strnad, E. Prinsen, Purification of 3-indolylacetic acid by solid phase extraction, J. Sep. Sci. 28 (2005) 1370-1374.

[50] P.E. Staswick, B. Serban, M. Rowe, I. Tiryaki, M.T. Maldonado, M.C. Maldonado, W. Suza, Characterization of an Arabidopsis enzyme family that conjugates amino acids to indole-3-acetic acid, Plant Cell. 17 (2005) 616-627.

[51] V.B. Tognetti, O. Van Aken, K. Morreel, K. Vandenbroucke, B. Van De Cotte, I. De Clercq, S. Chiwocha, R. Fenske, E. Prinsen, W. Boerjan, B. Genty, K.A. Stubbs, D. Inzé, F. Van Breusegem, Perturbation of indole-3-butyric acid homeostasis by the UDP-glucosyltransferase UGT74E2 modulates Arabidopsis architecture and water stress tolerance, Plant Cell. 22 (2010) 2660-2679.

[52] P.E. Staswick, The tryptophan conjugates of jasmonic and indole-3-acetic acids are endogenous auxin inhibitors, Plant Physiol. 150 (2009) 1310-1321.

[53] M. Kojima, T. Kamada-Nobusada, H. Komatsu, K. Takei, T. Kuroha, M. Mizutani, M. Ashikari, M. Ueguchi-Tanaka, M. Matsuoka, K. Suzuki, H. Sakakibara, Highly sensitive and high-throughput analysis of plant hormones using MS-probe modification and liquid chromatography-tandem mass spectrometry: an application for hormone profiling in Oryza sativa, Plant Cell Physiol. 50 (2009) 1201-1214.

[54] P.I. Dobrev, M. Kamínek, Fast and efficient separation of cytokinins from auxin and abscisic acid and their purification using mixed-mode solid-phase extraction, J. Chromatogr. A. 950 (2002) 2129.

[55] J. Mravec, P. Skůpa, A. Bailly, K. Hoyerová, P. Křeček, A. Bielach, J. Petrášek, J. Zhang, V. Gaykova, Y.-D. Stierhof, P.I. Dobrev, K. Schwarzerová, J. Rolčík, D. Seifertová, C. Luschnig, E. Benková, E. Zažímalová, M. Geisler, J. Friml, Subcellular homeostasis of phytohormone auxin is mediated by the ER-localized PIN5 transporter, Nature. 459 (2009) 1136-1140.

[56] P. Herrero, F. Borrull, E. Pocurull, R. Marcé, Efficient tandem solid-phase extraction and liquid chromatography-triple quadrupole mass spectrometry method to determine polar benzotriazole, benzothiazole and benzenesulfonamide contaminants in environmental water samples, $\mathrm{J}$. Chromatogr. A. 1309 (2013) 22-32.

[57] J. Laaks, M.A. Jochmann, T.C. Schmidt, Solvent-free microextraction techniques in gas chromatography, Anal. Bioanal. Chem. 402 (2012) 565-571. 
[58] R. Meyer, G.F. Rautenbach, I.A. Dubery, Identification and quantification of methyl jasmonate in leaf volatiles of Arabidopsis thaliana using solid-phase microextraction in combination with gas chromatography and mass spectrometry, Phytochem. Anal. 14 (2003) 155-159.

[59] M.L. Ruiz del Castillo, G.P. Blanch, Enantiomeric purity of (+/-)-methyl jasmonate in fresh leaf samples and commercial fragrances, J. Sep. Sci. 30 (2007) 2117-2122.

[60] C. Zadra, A. Borgogni, C. Marucchini, Quantification of jasmonic acid by SPME in tomato plants stressed by ozone, J. Agr. Food Chem. 54 (2006) 9317-9321.

[61] Q. Wu, D. Wu, Y. Guan, Polyaniline sheathed electrospun nanofiber bar for in vivo extraction of trace acidic phytohormones in plant tissue, J. Chromatogr. A. 1342 (2014) 16-23.

[62] C. Chen, Y. Chen, J. Zhou, C. Wu, A 9-vinyladenine-based molecularly imprinted polymeric membrane for the efficient recognition of plant hormone $1 \mathrm{H}$-indole-3-acetic acid, Anal. Chim. Acta. 569 (2006) 58-65.

[63] E. Turiel, A. Martin-Esteban, Molecularly imprinted polymers for sample preparation: a review, Anal. Chim. Acta. 668 (2010) 87-99.

[64] A. Beltran, F. Borrull, R. Marcé, P. Cormack, Molecularly-imprinted polymers: useful sorbents for selective extractions, TrAC Trends Anal. Chem. 29 (2010) 1363-1375.

[65] R. Garcia, M.J. Cabrita, A.M.C. Freitas, Application of molecularly imprinted polymers for the analysis of pesticide residues in food-A highly selective and innovative approach, American $\mathrm{J}$. Anal. Chem. 2 (2011) 16.

[66] N. Martins, E.P. Carreiro, M. Simões, M.J. Cabrita, A.J. Burke, R. Garcia, An emerging approach for the targeting analysis of dimethoate in olive oil: The role of molecularly imprinted polymers based on photo-iniferter induced "living" radical polymerization, React. Funct. Polym. 86 (2015) $37-46$.

[67] M. Simões, N. Martins, M.J. Cabrita, A.J. Burke, R. Garcia, Tailor-made molecularly imprinted polymers for dimethoate and deltamethrin recognition: synthesis, characterization and chromatographic evaluation, J. Polym. Res. 21 (2014) 1-13.

[68] H. Yan, F. Wang, D. Han, G. Yang, Simultaneous determination of four plant hormones in bananas by molecularly imprinted solid-phase extraction coupled with high performance liquid chromatography, Analyst. 137 (2012) 2884-2890.

[69] Y. Zhang, Y. Li, Y. Hu, G. Li, Y. Chen, Preparation of magnetic indole-3-acetic acid imprinted polymer beads with 4 -vinylpyridine and $\beta$-cyclodextrin as binary monomer via microwave heating initiated polymerization and their application to trace analysis of auxins in plant tissues, J. Chromatogr. A. 1217 (2010) 7337-7344.

[70] J.-F. Liu, J. Ding, B.-F. Yuan, Y.-Q. Feng, Magnetic solid phase extraction coupled with in situ derivatization for the highly sensitive determination of acidic phytohormones in rice leaves by UPLC-MS/MS, Analyst. 139 (2014) 5605-5613.

[71] B.-D. Cai, J. Yin, Y.-H. Hao, Y.-N. Li, B.-F. Yuan, Y.-Q. Feng, Profiling of phytohormones in rice under elevated cadmium concentration levels by magnetic solid-phase extraction coupled with liquid chromatography tandem mass spectrometry, J. Chromatogr. A. 1406 (2015) 78-86.

[72] M.-C. Hennion, V. Pichon, Immuno-based sample preparation for trace analysis, J. Chromatogr. A. 1000 (2003) 29-52.

[73] A.C. Moser, D.S. Hage, Immunoaffinity chromatography: an introduction to applications and recent developments, Bioanalysis. 2 (2010) 769-790.

[74] A. Pěnčík, J. Rolčík, O. Novák, V. Magnus, P. Barták, R. Buchtík, B. Salopek-Sondi, M. Strnada, Isolation of novel indole-3-acetic acid conjugates by immunoaffinity extraction, Talanta. 80 (2009) $651-655$.

[75] C.-S. Feung, R.H. Hamilton, R.O. Mumma, Metabolism of Indole-3-Acetic Acid III. Identification of Metabolites Isolated from Crown Gall Callus Tissue, Plant Physiol. 58 (1976) 666-669. 
[76] W.A. Stirk, G.D. Arthur, A. Lourens, O. Novák, M. Strnad, J. Van Staden, Changes in cytokinin and auxin concentrations in seaweed concentrates when stored at an elevated temperature, J. Appl. Phycol. 16 (2004) 31-39.

[77] D. Veselov, M. Langhans, W. Hartung, R. Aloni, I. Feussner, C. Götz, S. Veselova, S. Schlomski, C. Dickler, K. Bächmann, C.I. Ullrich, Development of Agrobacterium tumefaciens C58-induced plant tumors and impact on host shoots are controlled by a cascade of jasmonic acid, auxin, cytokinin, ethylene and abscisic acid, Planta. 216 (2003) 512-522.

[78] E.A. Schmelz, J. Engelberth, H.T. Alborn, P. O'Donnell, M. Sammons, H. Toshima, J.H. Tumlinson, Simultaneous analysis of phytohormones, phytotoxins, and volatile organic compounds in plants, Proc. Natl. Acad. Sci. 100 (2003) 10552-10557.

[79] X.-B. Yin, J.-M. Guo, W. Wei, Dual-cloud point extraction and tertiary amine labeling for selective and sensitive capillary electrophoresis-electrochemiluminescent detection of auxins, $\mathrm{J}$. Chromatogr. A. 1217 (2010) 1399-1406.

[80] Y.Y. Tam, E. Epstein, J. Normanly, Characterization of auxin conjugates in Arabidopsis. Low steady-state levels of indole-3-acetyl-aspartate, indole-3-acetyl-glutamate, and indole-3-acetylglucose, Plant Physiol. 123 (2000) 589-596.

[81] P. Picciarelli, N. Ceccarelli, F. Paolicchi, G. Calistri, Endogenous auxins and embryogenesis in Phaseolus coccineus, Funct. Plant Biol. 28 (2001) 73-78.

[82] D.E. Brown, A.M. Rashotte, A.S. Murphy, J. Normanly, B.W. Tague, W.A. Peer, L. Taiz, G.K. Muday, Flavonoids act as negative regulators of auxin transport in vivo in Arabidopsis, Plant Physiol. 126 (2001) 524-535.

[83] J. Ludwig-Müller, J.D. Cohen, Identification and quantification of three active auxins in different tissues of Tropaeolum majus, Physiol. Plantarum. 115 (2002) 320-329.

[84] S. Chiwocha, P. von Aderkas, Endogenous levels of free and conjugated forms of auxin, cytokinins and abscisic acid during seed development in Douglas fir, Plant Growth Regul. 36 (2002) 191-200.

[85] D.M. Ribnicky, J.D. Cohen, W.-S. Hu, T.J. Cooke, An auxin surge following fertilization in carrots: a mechanism for regulating plant totipotency, Planta. 214 (2002) 505-509.

[86] C.E. Jager, G.M. Symons, J.J. Ross, J.J. Smith, J.B. Reid, The brassinosteroid growth response in pea is not mediated by changes in gibberellin content, Planta. 221 (2005) 141-148.

[87] C. Solfanelli, S. Bartolini, C. Vitagliano, R. Lorenzi, Immunolocalization and quantification of IAA after self-and free-pollination in Olea europaea L., Sci. Hortic. 110 (2006) 345-351.

[88] M.I.A. Flores, R. Romero-González, A.G. Frenich, J.L.M. Vidal, QuEChERS-based extraction procedure for multifamily analysis of phytohormones in vegetables by UHPLC-MS/MS, J. Sep. Sci. 34 (2011) 1517-1524.

[89] J. Waterval, H. Lingeman, A. Bult, W.J. Underberg, Derivatization trends in capillary electrophoresis, Electrophoresis. 21 (2000) 4029-4045.

[90] S.D. Maleknia, K.M. Downard, Radical approaches to probe protein structure, folding, and interactions by mass spectrometry, Mass Spectrom. Review. 20 (2001) 388-401.

[91] W.-C. Yang, H. Mirzaei, X. Liu, F.E. Regnier, Enhancement of amino acid detection and quantification by electrospray ionization mass spectrometry, Anal. Chem. 78 (2006) 4702-4708. doi:10.1021/ac0600510.

[92] M. Eggink, M. Wijtmans, R. Ekkebus, H. Lingeman, I.J. de Esch, J. Kool, W.M.A. Niessen, H. Irth, Development of a selective ESI-MS derivatization reagent: synthesis and optimization for the analysis of aldehydes in biological mixtures, Anal. Chem. 80 (2008) 9042-9051. doi:10.1021/ac801429w.

[93] E. Prinsen, W. Van Dongen, E.L. Esmans, H.A. Van Onckelen, HPLC Linked Electrospray Tandem Mass Spectrometry: A Rapid and Reliable Method to Analyse Indole-3-Acetic Acid Metabolism in Bacteria, J. Mass Spectrom. 32 (1997) 12-22. 
[94] C. Schummer, O. Delhomme, B.M. Appenzeller, R. Wennig, M. Millet, Comparison of MTBSTFA and BSTFA in derivatization reactions of polar compounds prior to GC/MS analysis, Talanta. 77 (2009) 1473-1482.

[95] J. Chamarro, A. Östin, G. Sandberg, Metabolism of indole-3-acetic acid by orange (Citrus sinensis) flavedo tissue during fruit development, Phytochemistry. 57 (2001) 179-187.

[96] H. Chen, X.-F. Guo, H.-S. Zhang, H. Wang, Simultaneous determination of phytohormones containing carboxyl in crude extracts of fruit samples based on chemical derivatization by capillary electrophoresis with laser-induced fluorescence detection, J. Chromatogr. B. 879 (2011) 18021808.

[97] H. Chen, Z.-X. Zhang, G.-M. Zhang, X.-F. Guo, H.-S. Zhang, H. Wang, Liquid chromatographic determination of endogenous phytohormones in vegetable samples based on chemical derivatization with 6-oxy (acetylpiperazine) fluorescein, J. Agr. Food Chem. 58 (2010) 4560-4564.

[98] M.-L. Chen, Y.-Q. Huang, J.-Q. Liu, B.-F. Yuan, Y.-Q. Feng, Highly sensitive profiling assay of acidic plant hormones using a novel mass probe by capillary electrophoresis-time of flight-mass spectrometry, J. Chromatogr. B. 879 (2011) 938-944.

[99] E. Weiler, Immunoassay of plant growth regulators, Ann. Rev. Plant Physiol. 35 (1984) 85-95.

[100] B.-F. Liu, X.-H. Zhong, Y.-T. Lu, Analysis of plant hormones in tobacco flowers by micellar electrokinetic capillary chromatography coupled with on-line large volume sample stacking, J. Chromatogr. A. 945 (2002) 257-265.

[101] S. Wang, L. Jia, D. Xing, D. Chen, J. Zhao, On-line concentration and pressurized capillary electrochromatographic analysis of phytohormones in corn, J. Sep. Sci. 31 (2008) 859-864.

[102] Q. Lu, L. Zhang, L. Chen, M. Lu, P. Tong, G. Chen, Simultaneous analysis of endogenetic and ectogenic plant hormones by pressurized capillary electrochromatography, J. Sep. Sci. 33 (2010) 651-657.

[103] A. Segura Carretero, C. Cruces-Blanco, M. Soriano Peña, S. Cortacero Ramirez, A. Fernández Gutiérrez, Determination of phytohormones of environmental impact by capillary zone electrophoresis, J. Agr. Food Chem. 52 (2004) 1419-1422.

[104] N.A. Assunção, S.C. Arruda, A.P. Martinelli, E. Carrilho, Direct determination of plant-growth related metabolites by capillary electrophoresis with spectrophotometric UV detection, J. Brazil. Chem. Soc. 20 (2009) 183-187.

[105] C. Wei, H. Zhou, C. Chen, Z. Li, J. Zhou, On-Line Monitoring 1H-Indole-3-Acetic Acid in Plant Tissues Using Molecular Imprinting Monolayer Techniques on a Surface Plasmon Resonance Sensor, Anal. Lett. 44 (2011) 2911-2921.

[106] J.D. Cohen, B.G. Baldi, J.P. Slovin, 13C6-[Benzene Ring]-Indole-3-Acetic Acid A New Internal Standard for Quantitative Mass Spectral Analysis of Indole-3-Acetic Acid in Plants, Plant Physiol. 80 (1986) 14-19.

[107] A. Edlund, S. Eklof, B. Sundberg, T. Moritz, G. Sandberg, A microscale technique for gas chromatography-mass spectrometry measurements of picogram amounts of indole-3-acetic acid in plant tissues, Plant Physiol. 108 (1995) 1043-1047.

[108] T. Moritz, J.E. Olsen, Comparison between high-resolution selected ion monitoring, selected reaction monitoring, and four-sector tandem mass spectrometry in quantitative analysis of gibberellins in milligram amounts of plant tissue., Anal. Chem. 67 (1995) 1711-1716.

[109] D.H. Russell, R.D. Edmondson, High-resolution mass spectrometry and accurate mass measurements with emphasis on the characterization of peptides and proteins by matrix-assisted laser desorption/ionization time-of-flight mass spectrometry, J. Mass Spectrom. 32 (1997) 263276.

[110] Q. Lu, L. Zhang, T. Chen, M. Lu, T. Ping, G. Chen, Identification and quantitation of auxins in plants by liquid chromatography/electrospray ionization ion trap mass spectrometry, Rapid Commun. Mass Sp. 22 (2008) 2565-2572. 
[111] Y. Izumi, A. Okazawa, T. Bamba, A. Kobayashi, E. Fukusaki, Development of a method for comprehensive and quantitative analysis of plant hormones by highly sensitive nanoflow liquid chromatography-electrospray ionization-ion trap mass spectrometry, Anal. Chim. Acta. 648 (2009) 215-225.

[112] P.I. Dobrev, L. Havlíček, M. Vágner, J. Malbeck, M. Kamínek, Purification and determination of plant hormones auxin and abscisic acid using solid phase extraction and two-dimensional high performance liquid chromatography, J. Chromatogr. A. 1075 (2005) 159-166.

[113] N.S. Kiran, L. Polanská, R. Fohlerová, P. Mazura, M. Válková, M. Šmeral, J. Zouhar, J. Malbeck, P.I. Dobrev, I. Macháčová, B. Brzobohatý, Ectopic over-expression of the maize $\beta$-glucosidase Zm-p60. 1 perturbs cytokinin homeostasis in transgenic tobacco, J. Exp. Bot. 57 (2006) 985-996.

[114] L. Polanská, A. Vičánková, M. Nováková, J. Malbeck, P.I. Dobrev, B. Brzobohatý, R. Vaňková, I. Macháčová, Altered cytokinin metabolism affects cytokinin, auxin, and abscisic acid contents in leaves and chloroplasts, and chloroplast ultrastructure in transgenic tobacco, J. Exp. Bot. 58 (2007) 637-649.

[115] D.R. Stoll, J.D. Cohen, P.W. Carr, Fast, comprehensive online two-dimensional high performance liquid chromatography through the use of high temperature ultra-fast gradient elution reversedphase liquid chromatography, J. Chromatogr. A. 1122 (2006) 123-137.

[116] D.R. Stoll, X. Li, X. Wang, P.W. Carr, S.E.G. Porter, S.C. Rutan, Fast, comprehensive twodimensional liquid chromatography., J Chromatogr. A. 1168 (2007) 3-43; discussion 2.

[117] P. Dugo, F. Cacciola, T. Kumm, G. Dugo, L. Mondello, Comprehensive multidimensional liquid chromatography: theory and applications, J. Chromatogr. A. 1184 (2008) 353-368.

[118] J.B. Phillips, Z. Liu, US Patent No. 5,135,549, (1992).

[119] Z. Liu, J.B. Phillips, Comprehensive two-dimensional gas chromatography using an on-column thermal modulator interface, J. Chromatogr. Sci. 29 (1991) 227-231.

[120] P. Yu, New analytical methodologies in the study of auxin biochemistry, 2014.

[121] T. Wu, Y. Liang, X. Zhu, M. Zhao, H. Liu, Separation and quantification of four isomers of indole-3acetyl-myo-inositol in plant tissues using high-performance liquid chromatography coupled with quadrupole time-of-flight tandem mass spectrometry, Anal. Bioanal. Chem. 406 (2014) 32393247.

[122] X.-B. Yin, D.-Y. Liu, Polydopamine-based permanent coating capillary electrochromatography for auxin determination, J. Chromatogr. A. 1212 (2008) 130-136.

[123] X.-B. Yin, E. Wang, Capillary electrophoresis coupling with electrochemiluminescence detection: a review, Anal. Chim. Acta. 533 (2005) 113-120.

[124] A. Varenne, S. Descroix, Recent strategies to improve resolution in capillary electrophoresis-A review, Anal. Chim. Acta. 628 (2008) 9-23.

[125] Z. Xi, Z. Zhang, Y. Sun, Z. Shi, W. Tian, Determination of indole-3-acetic acid and indole-3-butyric acid in mung bean sprouts using high performance liquid chromatography with immobilized $\mathrm{Ru}$ (bpy) 3 2+-KMnO 4 chemiluminescence detection, Talanta. 79 (2009) 216-221.

[126] S. Fuchs, J. Haimovich, Y. Fuchs, Immunological studies of plant hormones, Eur. J. Biochem. 18 (1971) 384-390.

[127] J. Swaczynová, O. Novák, E. Hauserová, K. Fuksová, M. Šíša, L. Kohout, M. Strnad, New techniques for the estimation of naturally occurring brassinosteroids, J. Plant Growth Regul. 26 (2007) 1-14.

[128] C. Bianco, R. Defez, Medicago truncatula improves salt tolerance when nodulated by an indole-3acetic acid-overproducing Sinorhizobium meliloti strain, J. Exp. Bot. 60 (2009) 3097-3107.

[129] Q. Chen, W. Qi, R.J. Reiter, W. Wei, B. Wang, Exogenously applied melatonin stimulates root growth and raises endogenous indoleacetic acid in roots of etiolated seedlings of Brassica juncea, J. Plant Physiol. 166 (2009) 324-328. 
[130] A. Sadanandom, R.M. Napier, Biosensors in plants, Curr. Opin. Plant Biol. 13 (2010) 736-743.

[131] C. Moina, G. Ybarra, Fundamentals and applications of immunosensors, in: Advances in Immunoassay Technology, Dr. Norman H. L. Chiu (Ed.), ISBN: 978-953-51-0440-7, InTech, DOI: 10.5772/36947. Available from: http://www.intechopen.com/books/advances-in-immunoassaytechnology/fundamentals-and-applications-of-immunosensors.

[132] J. Li, Z.-Y. Wu, L.-T. Xiao, G.-M. Zeng, G.-H. Huang, G.-L. Shen, R.-Q. Yu, A Novel Piezoelectric Biosensor for the Detection of Phytohormone. BETA.-Indole Acetic Acid., Anal. Sci. 18 (2002) 403-407.

[133] J. Li, L.-T. Xiao, G.-M. Zeng, G.-H. Huang, G.-L. Shen, R.-Q. Yu, A renewable amperometric immunosensor for phytohormone $\beta$-indole acetic acid assay, Anal. Chim. Acta. 494 (2003) 177185.

[134] S. Mancuso, A.M. Marras, V. Magnus, F. Balulvska, Noninvasive and continuous recordings of auxin fluxes in intact root apex with a carbon nanotube-modified and self-referencing microelectrode, Anal. Biochem. 341 (2005) 344-351.

[135] P. Hernandez, F. Galan, O. Nieto, L. Hernandez, Direct determination of indole-3-acetic acid in plant tissues by electroanalytical techniques using a carbon paste modified with OV-17 electrode, Electroanalysis. 6 (1994) 577-583.

[136] E.S. McLamore, A. Diggs, P. Calvo Marzal, J. Shi, J.J. Blakeslee, W.A. Peer, A.S. Murphy, D.M. Porterfield, Non-invasive quantification of endogenous root auxin transport using an integrated flux microsensor technique, Plant J. 63 (2010) 1004-1016.

[137] K. Wu, Y. Sun, S. Hu, Development of an amperometric indole-3-acetic acid sensor based on carbon nanotubes film coated glassy carbon electrode, Sensor. Actuat. B-Chem. 96 (2003) 658662.

[138] Y. Zhou, Z. Xu, M. Wang, X. Meng, H. Yin, Electrochemical immunoassay platform for high sensitivity detection of indole-3-acetic acid, Electrochim. Acta. 96 (2013) 66-73.

[139] H. Yin, Z. Xu, Y. Zhou, M. Wang, S. Ai, An ultrasensitive electrochemical immunosensor platform with double signal amplification for indole-3-acetic acid determinations in plant seeds, Analyst. 138 (2013) 1851-1857.

[140] B. Sun, L. Chen, Y. Xu, M. Liu, H. Yin, S. Ai, Ultrasensitive photoelectrochemical immunoassay of indole-3-acetic acid based on the MPA modified CdS/RGO nanocomposites decorated ITO electrode, Biosens. Bioelectron. 51 (2014) 164-169.

[141] Y.J. Yang, X. Xiong, K. Hou, S. Hu, The amperometric determination of indole-3-acetic acid based on CeCl3-DHP film modified gold electrode, Russ. J. Electrochem. 47 (2011) 47-52.

[142] S. Zhang, K. Wu, Square wave voltammetric determination of indole-3-acetic acid based on the enhancement effect of anionic surfactant at the carbon paste electrode, Bull. Korean Chem. Soc. 25 (2004) 1321-1325.

[143] S. Nag, K. Saha, M. Choudhuri, Role of auxin and polyamines in adventitious root formation in relation to changes in compounds involved in rooting, J. Plant Growth Regul. 20 (2001) 182-194.

[144] S. Nag, A. Paul, M. Choudhuri, Changes In Peroxidase Activity During Adventitious Root Formation At The Base Of Mung Bean Cuttings, Int. J. Sci. Tech. Res. 2 (2013) 171-179.

[145] J.-M. Guo, Y.-Y. Xin, X.-B. Yin, Selective differentiation of indoleacetic acid and indolebutyric acid using colorimetric recognition after Ehrlich reaction, J. Agr. Food Chem. 58 (2010) 6556-6561.

[146] P. Pattnaik, Surface plasmon resonance, Appl. Biochem. Biotechnol. 126 (2005) 79-92.

[147] J. Homola, Present and future of surface plasmon resonance biosensors, Anal. Bioanal. Chem. 377 (2003) 528-539.

[148] M. Stoeckli, P. Chaurand, D.E. Hallahan, R.M. Caprioli, Imaging mass spectrometry: a new technology for the analysis of protein expression in mammalian tissues, Nat. Med. 7 (2001) 493496. 
[149] J.H. Jun, Z. Song, Z. Liu, B.J. Nikolau, E.S. Yeung, Y.J. Lee, High-spatial and high-mass resolution imaging of surface metabolites of Arabidopsis thaliana by laser desorption-ionization mass spectrometry using colloidal silver, Anal. Chem. 82 (2010) 3255-3265.

[150] K. Takahashi, T. Kozuka, A. Anegawa, A. Nagatani, T. Mimura, Development and Application of a High-Resolution Imaging Mass Spectrometer for the Study of Plant Tissues, Plant Cell Physiol. (2015) pcv083.

[151] G. Ouyang, D. Vuckovic, J. Pawliszyn, Nondestructive sampling of living systems using in vivo solid-phase microextraction, Chem. Rev. 111 (2011) 2784-2814.

[152] C. Duan, Z. Shen, D. Wu, Y. Guan, Recent developments in solid-phase microextraction for onsite sampling and sample preparation, TrAC Trends Anal. Chem. 30 (2011) 1568-1574.

[153] X. Hoa, A. Kirk, M. Tabrizian, Towards integrated and sensitive surface plasmon resonance biosensors: a review of recent progress, Biosens. Bioelectron. 23 (2007) 151-160.

[154] K.M. Mayer, J.H. Hafner, Localized surface plasmon resonance sensors, Chem. Rev. 111 (2011) 3828-3857.

[155] M. Careri, A. Mangia, Trends in analytical atomic and molecular mass spectrometry in biology and the life sciences, Anal. Bioanal. Chem. 399 (2011) 2585-2595. 


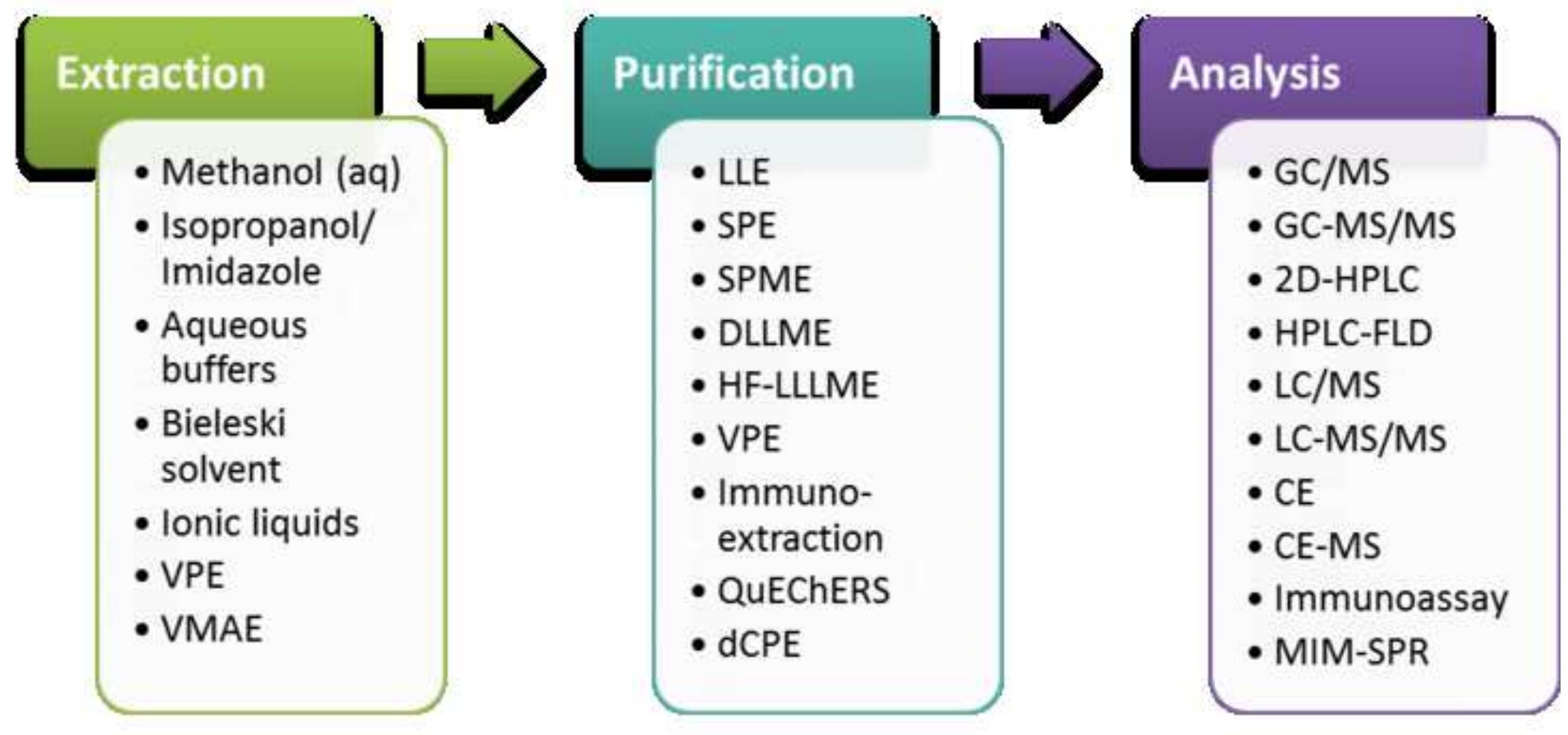

- Methanol (aq) Imidazole

Aqueous buffers

- Bieleski solvent

- Ionic liquids

- VPE

- VMAE
- $\mathrm{GC} / \mathrm{MS}$

GC-MS/MS

2D-HPLC

- HPLC-FLD

LC/MS

LC-MS/MS

- CE

- Immunoassay

MIM-SPR 


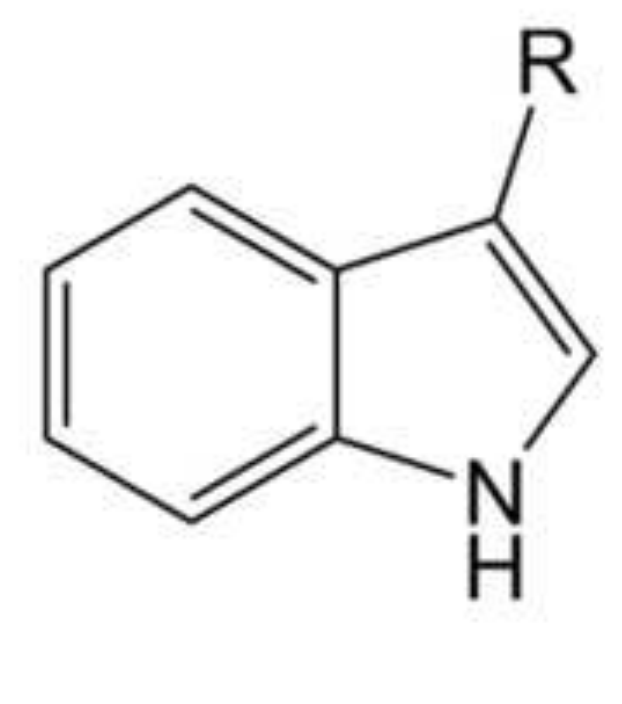

R

$\mathrm{COOH}$ $\mathrm{CH}_{2} \mathrm{COOH}$

$\mathrm{CH}_{2} \mathrm{CH}_{2} \mathrm{COOH}$
$\mathrm{CH}_{2} \mathrm{CH}_{2} \mathrm{CH}_{2} \mathrm{COOH}$

$\mathrm{CH}_{2} \mathrm{CH}_{2} \mathrm{COOH}$
$\mathrm{CH}_{2} \mathrm{CH}_{2} \mathrm{CH}_{2} \mathrm{COOH}$

$\mathrm{CH}_{2} \mathrm{COOHCH}_{3}$

R

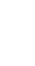
Auxin
ICA - indole-3-carboxylic acid IAA - indole-3-acetic acid
IPA - indole-3-propionic acid IBA - indole-3-butyric acid MelAA - IAA methyl ester
Auxin

(n)

\begin{abstract}
(
\end{abstract}

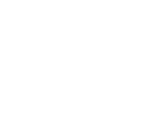

(n)



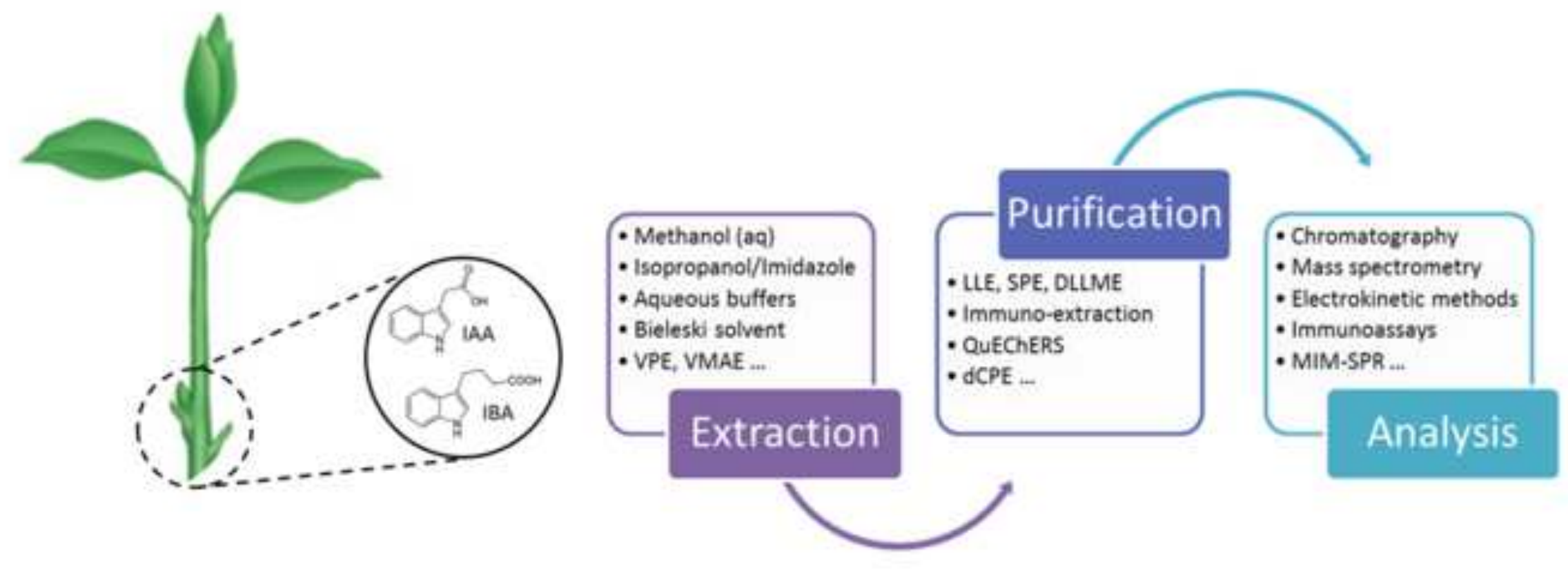